OPEN ACCESS

Edited by:

Aiping Lu,

Hong Kong Baptist University,

Hong Kong

Reviewed by:

Zhenhua Dai,

Guangdong Provincial Academy of

Chinese Medical Sciences, China

Thomas Heinbockel,

Howard University, United States

*Correspondence:

Jie Wang

wangjiedoctor2015@163.com

${ }^{\dagger}$ These authors have contributed equally to this work.

Specialty section:

This article was submitted to Ethnopharmacology,

a section of the journal

Frontiers in Pharmacology

Received: 20 July 2017 Accepted: 20 September 2017 Published: 17 October 2017

Citation:

Duan L, Xiong X, Hu J, Liu Y, Li J and

Wang J (2017) Panax notoginseng Saponins for Treating Coronary Artery Disease: A Functional and Mechanistic

Overview. Front. Pharmacol. 8:702. doi: 10.3389/fphar.2017.00702

\section{Panax notoginseng Saponins for Treating Coronary Artery Disease: A Functional and Mechanistic Overview}

\author{
Lian Duan ${ }^{1,2 \dagger}$, Xingjiang Xiong ${ }^{1 \dagger}$, Junyuan Hu${ }^{1,2}$, Yongmei $\mathrm{Liu}^{1}$, Jun $\mathrm{Li}^{1}$ and Jie Wang ${ }^{1 *}$ \\ ${ }^{1}$ Department of Cardiology, Guang'anmen Hospital, China Academy of Chinese Medical Science, Beijing, China, ${ }^{2}$ Graduate \\ School, Beijing University of Traditional Chinese Medicine, Beijing, China
}

Coronary artery disease (CAD) is a major public health problem and the chief cause of morbidity and mortality worldwide. Panax notoginseng, a valuable herb in traditional Chinese medicine (TCM) with obvious efficacy and favorable safety, shows a great promise as a novel option for CAD and is increasingly recognized clinically. Firstly, this review introduced recent clinical trials on treatment with PNS either alone or in combination with conventional drugs as novel treatment strategies. Then we discussed the mechanisms of $P$. notoginseng and Panax notoginseng saponins (PNS), which can regulate signaling pathways associated with inflammation, lipid metabolism, the coagulation system, apoptosis, angiogenesis, atherosclerosis, and myocardial ischaemia.

Keywords: Panax notoginseng, PNS, coronary artery disease, traditional Chinese medicine, review

\section{INTRODUCTION}

Coronary artery disease (CAD) is a major public health problem and a chief cause of morbidity and mortality worldwide. The number of deaths due to CAD was 56 million people globally during a decade from 2000 (WHO, 2014). The sum of hospitalized cardiovascular operations rised by $28 \%$, from about 6 to 7.5 million (Mozaffarian et al., 2016). The economic load of caring for patients also represents a huge cost for society. Revascularization represents innovative progress in the treatment for symptomatic CAD. However, by targeting one or two vascular lesions, it fails to completely solve the problem of plaque progress. A truly advance in the treatment of CAD will require more effective prevention. At the end of the nineteenth century, drug resistance to organic nitrates was observed (Dilidar, 2009; Münzel et al., 2011), while aspirin resistance was observed in 1994 (Helgason et al., 1994). Clopidogrel has been widely used in various thrombotic diseases, especially in CAD patients with percutaneous coronary intervention (PCI; O'Gara et al., 2013). About a quarter of patients administering standard loading of clopidogrel exhibit poor responsiveness (Serebruany et al., 2005). Due to the complexity of CAD, most patients require lifelong medication. Moreover, oral aspirin may directly stimulate the gastric mucosa and initiate abdominal discomfort, nausea and vomiting. The long-term use of aspirin can easily cause gastric mucosal damage (Hirsh et al., 1995). The risk of diabetes greatly increases with large doses of statins. In addition, statins can cause abnormal liver enzymes and myopathy (Thompson et al., 2016). Varying degrees of drug resistance and adverse reactions increase the difficulty and dissatisfaction with treatment.

In recent years, Traditional Chinese Medicine (TCM) has gained widespread popularity. Furthermore, an increasing number of studies have confirmed the efficacy of TCM for treating CAD. In 2007, nearly 4 out of 10 adults had used TCM therapy in the past 12 months, with 
natural products as the most commonly used therapies (Barnes et al., 2008). Of the various natural products, Panax notoginseng is one of the most commonly applied products because it has been evaluated with various beneficial effects, such as promotion of blood circulation, cerebrovascular protection (Liu L. et al., 2014; Zhang et al., 2016), improvement of neurological function (Gao et al., 2012), reduction of oxidative stress (Ding et al., 2015), and mitigation of bone loss (Fan et al., 2015).

Panax notoginseng is particularly popular among patients with CAD because many studies have been associated the consumption of $P$. notoginseng with CAD treatment. Panax notoginseng saponin (PNS) is the main active ingredient of $P$. notoginseng. Over the past over 40 years, great energy has been devoted to confirming the effectiveness of the compounds of $P$. notoginseng on CAD. Many animal experiments have shown that PNS can improve the energy metabolism of myocardial cells, reduce myocardial damage, and inhibit ischaemia-induced cardiomyocyte apoptosis in acute MI rats (Chen et al., 2011; Yue et al., 2012). Now the compound of PNS is available as an over-the-counter drug in both China and worldwide. In China alone, 5,000 tons of $P$. notoginseng products are produced annually (Liu J. et al., 2014). The role of P. notoginseng in cardiovascular diseases has been summarized (Liu J. et al., 2014; Yang et al., 2014), but no articles have focused on the effects of $P$. notoginseng against CAD. This review summarizes extensively recent evidence on the use of $P$. notoginseng in CAD therapy, its therapeutic effects and adverse events. Our current understanding of $P$. notoginseng cardioprotective effects and mechanisms against CAD will also be discussed in detail.

\section{METHODOLOGY}

The terms "P. notoginseng" or "Sanqi" or "P. notoginseng saponins" were searched as "Title/Abstract" and MeSH terms in PubMed, China National Knowledge Infrastructure (CNKI) and SinoMed database. Articles related to therapeutic effects in coronary artery disease (CAD) were picked out manually. All articles with abstract were included and no language restrictions was applied.

\section{BIOLOGICAL CHARACTERISTICS OF PANAX NOTOGINSENG}

\section{Brief History}

Panax notoginseng is a medicinal plant that was first used by ethnic minorities in China. It has been one of the most acclaimed herbs in China for 400 years. Panax notoginseng is traditionally applied as an anodyne and a hemorheologicaltering drug. The main medical component is the radix of $P$. notoginseng, also known as Sanqi, Tianqi, and Shanqi in East Asian countries (Wang T. et al., 2016). "Compendium of Materia Medica” (Bencao Gangmu 本草纲目) recorded the official detailed medical applications of $P$. notoginseng in 1758, in which $P$. notoginseng is called "more precious than gold” (jinbuhuan 金不换) and written as “三七” in Chinese (red box in Figure 1). As one species of ginseng, it was unrecognized worldwide until American ginseng was discovered in 1716. In 1843, the Russian botanist Carl Meyer nominate Asian ginseng the botanical name "Panax," which implies "all-healing" in Greek (Wong et al., 2015). "Noto" means "back, spine," and "ginseng" represents "essence of men.”

\section{Botanical Characteristics}

Panax notoginseng F.H. Chen is a hemitropous perennial herb with a short rhizome, a bamboo whip, and 2 to several fleshy roots (Editorial Board of Flora of China, 1978). The taproot looks conical or cylindrical with a length of $1-6 \mathrm{~cm}$ and a diameter of 1-4 cm. The surface is grayish brown or grayish yellow with intermittent vertical wrinkles and branch marks. There are stem scars on top with surrounding tumor-like bulges. The characteristics include dense, solid gray green, yellow green, or gray sections (Figure 2).

\section{PNS: The Major Therapeutic Constituents of Panax notoginseng}

Panax notoginseng contains three key constituents, including saponins, polysaccharides, and dencichine (Kim, 2012). Polysaccharides have many physiological functions, such as anti-tumor and immune regulation activity (Gu et al., 2015). A special type of amino acid, dencichine, is an active substance of $P$. notoginseng. Its structure is $\beta$-NoXalo-L- $\alpha, \beta$ diaminopropionic acid and can be artificially synthetized (Wei and Wang, 1987). Saponins constitute one of the most important effective components of $P$. notoginseng. So far, more than 80 kinds of monomer saponins have been isolated from $P$. notoginseng parts (radix, stem, leaf, alabastrum, seed, etc.) since their first separation and identification in 1979 (Wu, 1979). However, the most important components of PNS are ginsenoside Rg1, $\mathrm{Rb} 1, \mathrm{Re}$, and notoginsenoside R1 (NR1; Figure 3). The amounts of these four components in PNS are 30, 2.5, 20, and 2.5\%, respectively. The radix of $P$. notoginseng is the main part for extraction. According to the different aglycone structures, saponins are divided into two categories: dammarane tetracyclic triterpene and oleanane type pentacyclic triterpene. Based on whether the mother molecular nucleus structure of C6 displaces a hydroxyl group, dammarane-type saponins can be divided into protopanaxadiol (PPD), protopanaxatriol (PPT), or octotillol (Wong et al., 2015). Ginsenoside Rb1 is one of the major protopanaxadiol-type saponins. Ginsenoside Re, Rg1, and NR1 are the main raw ginseng triol saponins (Wong et al., 2015).

\section{Quality Control}

Many plant species are named Sanqi ( $P$. notoginseng). According to the statistics of the reference literature, except for Araliaceae $P$. notoginseng, medicinal plants named Sanqi ( $P$. notoginseng) include as many as 20 species belonging to 11 families, which causes difficulty in distinguishing real $P$. notoginseng. Pharmacological studies indicated that ginsenoside Rg1, ginsenoside Rb1, and NR1 are the main active constituents of $P$. notoginseng. The quality control of $P$. notoginseng also focuses on these three components. According to the "Chinese 

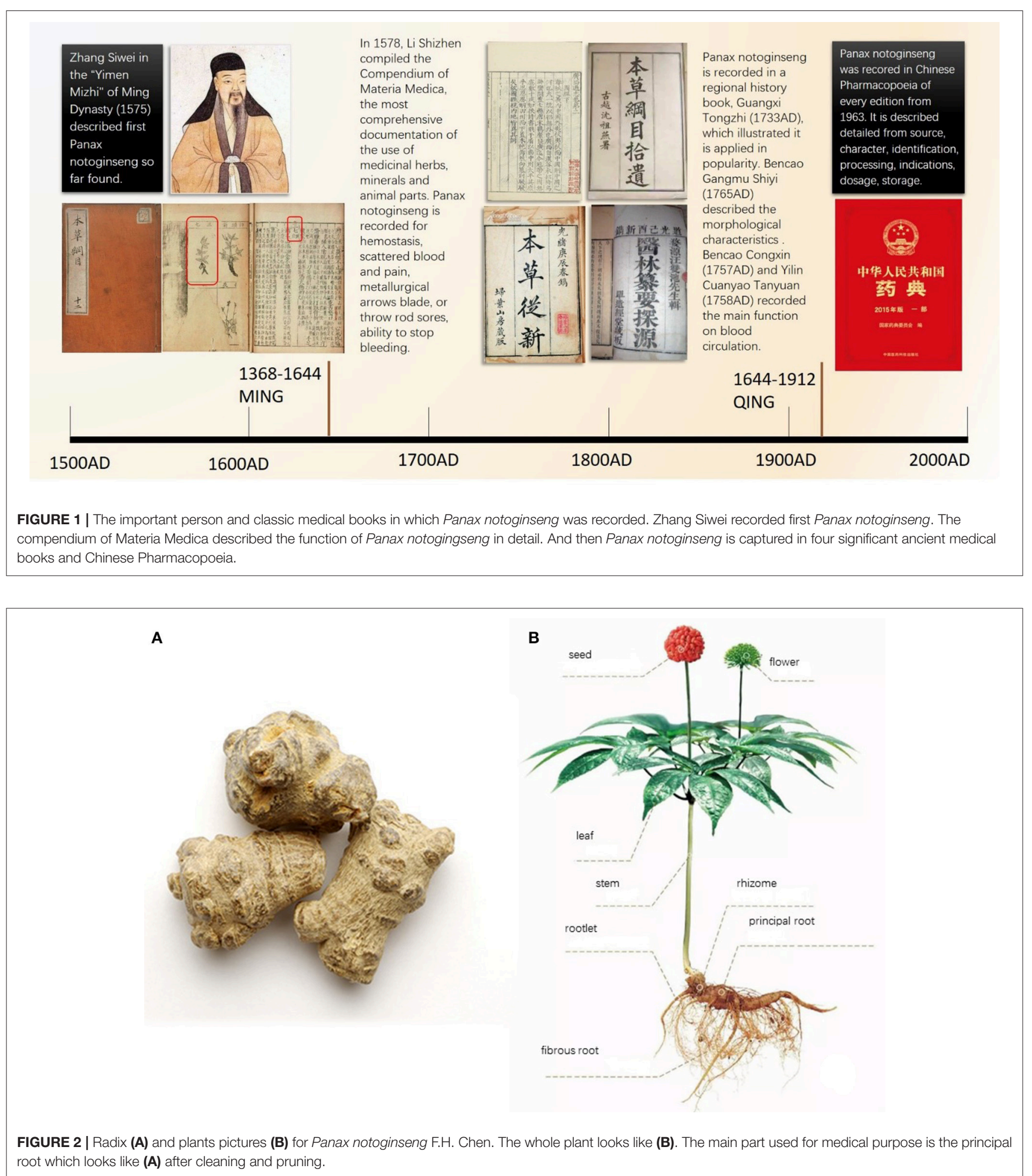

Pharmacopoeia," the distinction process is as follows: precisely extract the control solution and the test solution, pour into a liquid chromatograph, and determine the species. The total amounts of ginsenoside $\operatorname{Rg} 1$ (C42H72O14), ginsenoside
Rb1 (C54H92O23), and NR1 (C47H80O18) should not be $<5 \%$. In standard Chinese Medicinal Materials in Hongkong (HKCMMS, 2017), P. notoginseng can be identified by thin layer chromatography. The chromatographic results indicate 

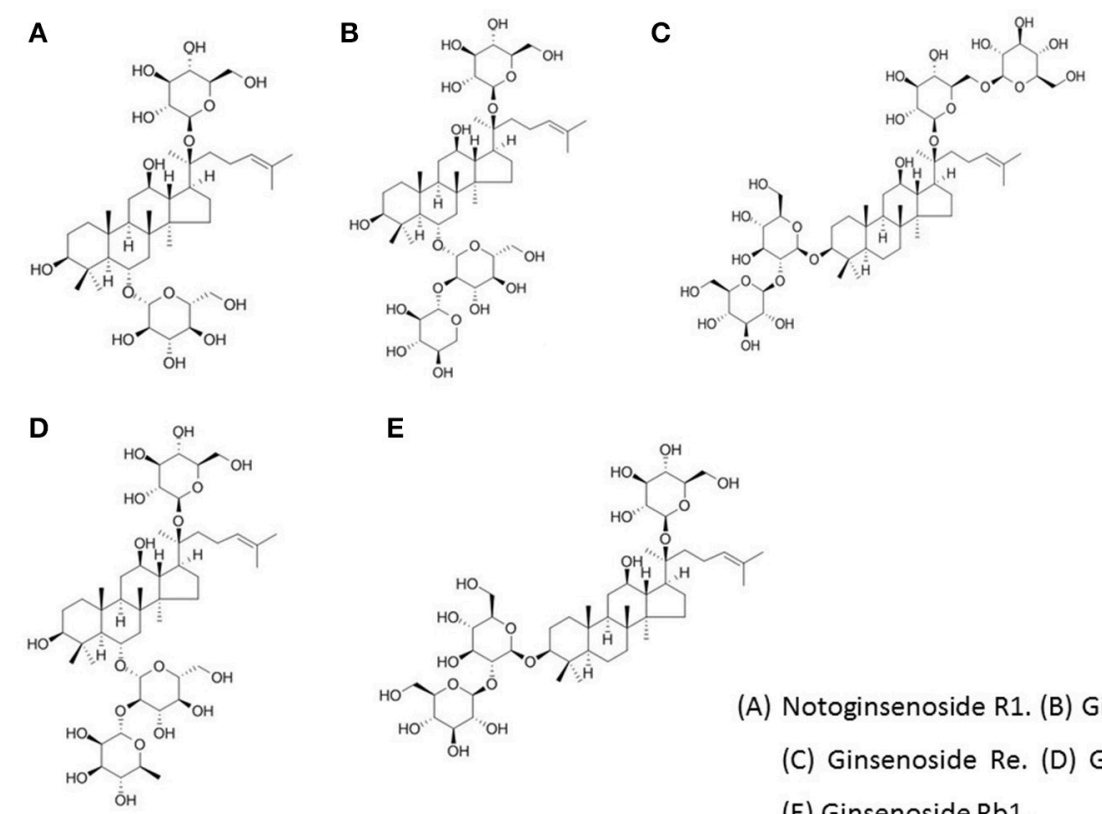

(A) Notoginsenoside R1. (B) Ginsenoside Rg1.

(C) Ginsenoside Re. (D) Ginsenoside Rd.

(E) Ginsenoside Rb1.

FIGURE 3 | The chemical structure of the main active ingredients of PNS. (A) Notoginsenoside R1. (B) Ginsenoside Rg1. (C) Ginsenoside Re. (D) Ginsenoside Rd. (E) Ginsenoside Rb1 ${ }^{1}$.

that the Rb1, Rg1, and NR1 have the same colors and Rf values. In addition, the relative retention time of $P$. notoginseng and the characteristic peaks of the six extraction liquids should be in accordance with the standard by HPLC fingerprint identification.

\section{THE THERAPEUTIC EFFECTS OF PNS ON CAD}

\section{Search Strategy}

We conducted a systematic search of oral PNS for over 4 weeks against $\mathrm{CAD}$ on four English databases and four Chinese databases: MEDLINE, the Cochrane Central Register of Controlled Trials (CENTRAL), EMBASE Database, WHO Clinical Trials Registration Platform, Chinese National Knowledge Infrastructure (CNKI), Chinese Scientific Journal Database (VIP), WANFANG, and SinoMed. The search time frame ranged from the databases' inception until 20 Feb 2017. We also searched reference lists for further publications. The search expression used in MEDLINE was ("coronary heart disease" [MeSH Terms] OR ("coronary artery disease" [MeSH Terms] AND ("P. notoginseng" [MeSH Terms] OR sanqi[Text Word] OR sanchi[Text Word])) OR Xuesaitong[Text Word] OR Xueshuantong[Text Word])) AND "Randomized Controlled Trial" [Publication Type:NoExp]. Similar expressions were used in the other databases. Outcome measures meet the primary or secondary outcomes.

${ }^{1}$ ChemFaces http://www.chemfaces.com/

\section{Study Quality}

Seventeen randomized clinical trials with 1,747 participants was collected which randomly assigned to a conventional treatment vs. a PN preparation evaluated cardiovascular outcomes (Table 1). The quality of the 17 RCTs was evaluated from seven aspects using the ROB scale in the Cochrane handbook (Table 2). Three RCTs indicated the random way as random numbers. However, the other studies didn't describe the random method. Two RCT (Han, 2008; Teng, 2014) refers to random concealment and blindness.

\section{Primary Outcome}

The primary outcome of CAD is the primary end point which was defined as the composite of all-cause mortality, myocardial infarction (MI), revascularization, and rehospitalization for unstable angina. PNS has been observed to have several beneficial effects in patients with different stages of CAD. Several RCTs reported oral PNS could reduce the primary outcome. In 2008, a team underwent a RCT of 60 patients with CAD after PCI. The patients who had PNS (120 mg every time, three times every day) or a placebo was prescribed daily in combination with their conventional therapy for 3 months. The end point, rehospitalization, was focused on. The rehospitalization rate of patients with PNS was better than in the control group (1/30 and 3/30; Han, 2008) (Table 3). In 2013, furthermore, another team conducted a 1-year RCT with $\sim 1,200$ CAD patents, 600 patients in the experimental group were given PNS (300 mg every time, three times every day). PNS increased the inhibitory effect of clopidogrel on platelet aggregation and reduced the primary end points. This trial compared the incidence of the primary end 


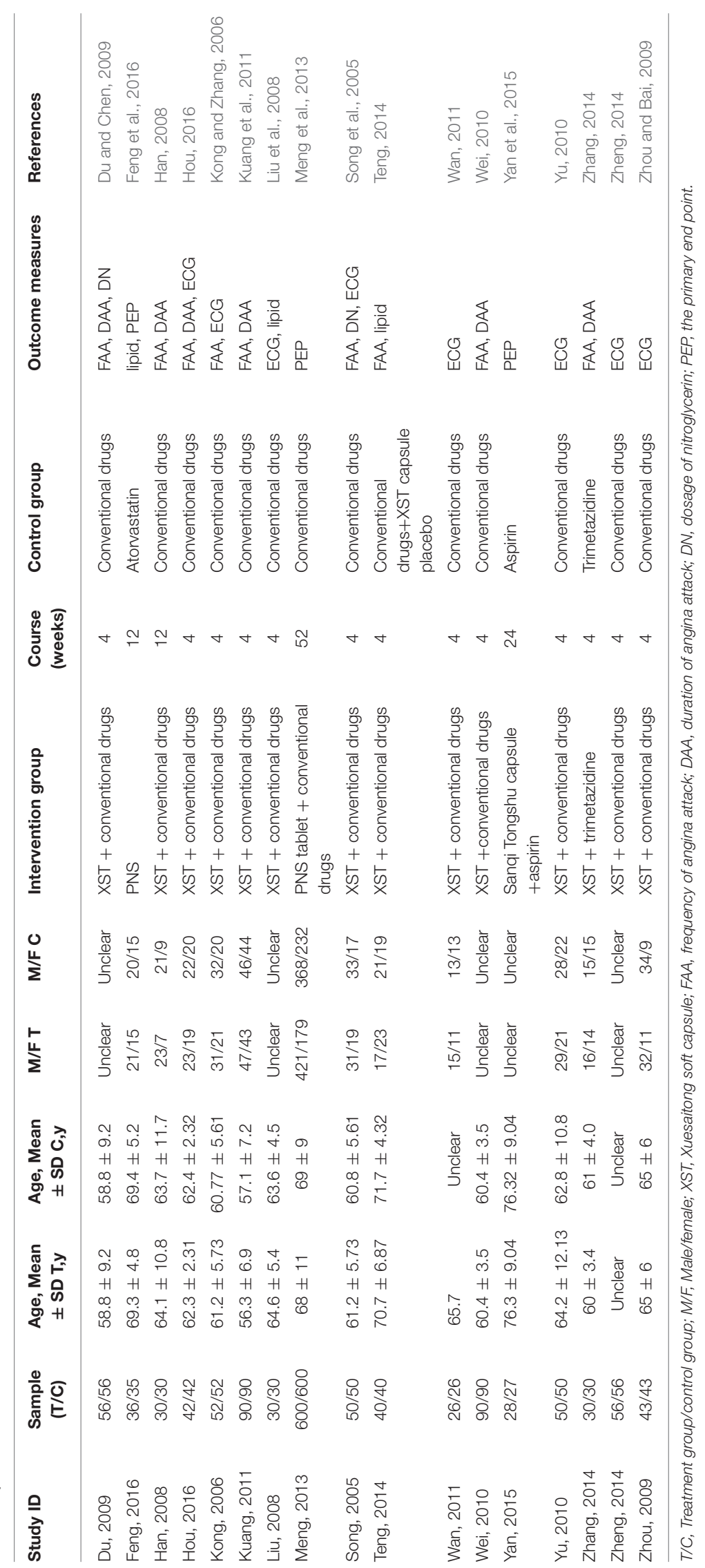


TABLE 2 | Risk of bias in the 17 RCTs of PNS on CAD.

\begin{tabular}{llllllll}
\hline References & A & B & C & D & E & F & G \\
\hline Du and Chen, 2009 & $?$ & $?$ & $?$ & $?$ & $?$ & $?$ & $?$ \\
Feng et al., 2016 & $?$ & $?$ & $?$ & $?$ & $?$ & $?$ & $?$ \\
Han, 2008 & + & + & + & + & & & \\
Hou, 2016 & $?$ & $?$ & $?$ & $?$ & $?$ & $?$ & $?$ \\
Kong and Zhang, 2006 & $?$ & $?$ & $?$ & $?$ & $?$ & $?$ & $?$ \\
Kuang et al., 2011 & $?$ & $?$ & $?$ & $?$ & $?$ & $?$ & $?$ \\
Liu et al., 2008 & + & + & + & $?$ & $?$ & $?$ & $?$ \\
Meng et al., 2013 & + & $?$ & $?$ & $?$ & $?$ & $?$ & $?$ \\
Song et al., 2005 & $?$ & $?$ & $?$ & $?$ & $?$ & $?$ & $?$ \\
Teng, 2014 & + & + & + & + & $?$ & $?$ & $?$ \\
Wan, 2011 & $?$ & $?$ & $?$ & $?$ & $?$ & $?$ & $?$ \\
Wei, 2010 & $?$ & $?$ & $?$ & $?$ & $?$ & $?$ & $?$ \\
Yan et al., 2015 & $?$ & $?$ & $?$ & $?$ & $?$ & $?$ & $?$ \\
Yu, 2010 & $?$ & $?$ & $?$ & $?$ & $?$ & $?$ & $?$ \\
Zhang, 2014 & $?$ & $?$ & $?$ & $?$ & $?$ & $?$ & $?$ \\
Zheng, 2014 & $?$ & $?$ & $?$ & $?$ & $?$ & $?$ & $?$ \\
Zhou and Bai, 2009 & $?$ & $?$ & $?$ & $?$ & $?$ & $?$ & $?$ \\
\hline ? & $?$
\end{tabular}

$A$, Random sequence generation; $B$, allocation concealment; $C$, blinding of participants and personnel; $D$, blinding for outcome assessment; $E$, incomplete outcome data; $F$, selective reporting; $G$, others bias; +, low risk of bias; -, high risk of bias; ?, unclear risk of bias.

TABLE 3 | The end point with PNS+conventional drugs and conventional drugs alone.

\begin{tabular}{lcccc}
\hline & & $\begin{array}{c}\text { PNS+conventional } \\
\text { drugs }\end{array}$ & $\begin{array}{c}\text { Conventional } \\
\text { drugs }\end{array}$ & $P$-value \\
\hline Cardiac death & $52 \mathrm{w}$ & $1 / 600$ & $1 / 600$ & $>0.05$ \\
& $12 \mathrm{w}$ & $0 / 30$ & $0 / 30$ & $>0.05$ \\
\hline Myocardial infarction & $52 \mathrm{w}$ & $2 / 600$ & $4 / 600$ & $<0.05$ \\
& $12 \mathrm{w}$ & $0 / 30$ & $0 / 30$ & $>0.05$ \\
\hline Revascularization & $52 \mathrm{w}$ & $16 / 600$ & $37 / 600$ & $<0.05$ \\
& $12 \mathrm{w}$ & $0 / 30$ & $0 / 30$ & $>0.05$ \\
\hline $\begin{array}{l}\text { Stent thrombosis } \\
\text { Rehospitalization for }\end{array}$ & $12 \mathrm{w}$ & $1 / 600$ & $5 / 600$ & $<0.05$ \\
\hline $\begin{array}{l}\text { Rnstable angina } \\
\text { Un }\end{array}$ & & $1 / 30$ & $3 / 30$ & $>0.05$ \\
\hline
\end{tabular}

points in the experiment and control groups, which was $3.3 \%$ (20 cases) and 7.8\% (47 cases; Meng et al., 2013), resepctively. The primary end points included cardiac death, myocardial infarction, revascularization, stent thrombosis, of which most were related to revascularization.

\section{Secondary Outcomes}

Secondary outcomes include electrocardiogram (ECG), attack of angina pectoris, such as frequency of angina pectoris, duration of angina pectoris and dosage of nitroglycerin, quality of life. Two systematic reviews estimated current evidence for the benefit of secondary outcomes and adverse events of PNS for
CAD. One systematic review included 17 randomized clinical trials. Oral PN could alleviate angina pectoris (Shang et al., 2013). Another systematic review including a total of six RCTs with 716 participants on unstable angina pectoris(UA) studied PNS alone or combined with conventional drugs vs. conventional drugs alone. The results illustrated that PNS combined with conventional drugs displayed also a significant effect on relieving angina symptoms and improving ECG compared with conventional drugs alone (Yang et al., 2013).

\section{Attack of Angina Pectoris}

Angina pectoris is the symptoms for chest pain or discomfort due to CAD (Xiong et al., 2015). The patients may also feel the discomfort in your neck, jaw, shoulder, back, or arm. Conventional drugs include anti-ischemic agents and vascular protective agents, such as nitroglycerin, aspirin, clopidogrel, betablockers, and statin (Smith et al., 2011).

In this overview, nine RCTs reviewed the therapeutic effects of PNS on angina pectoris compared PNS + conventional drugs with conventional drugs. It's demonstrated PNS is one effective agents to decrease frequency and duration of angina pectoris. PNS could decrease significantly frequency and duration of angina pectoris. 180 patients of unstable angina were randomly divided into treatment group and control group of, respectively 90 patients. The treatment group added PNS (2 times/d for 4 weeks) on the basis of conventional treatment of angina pectoris. The control group administered conventional treatment of angina pectoris. The results showed that the frequency of unstable angina pectoris, pain intensity and duration were significantly reduced (Kuang et al., 2011).

\section{Ischemic Changes on ECG}

ECG is the other important secondary outcome on evaluating the clinical efficacy against angina pectoris. A total of eight RCTs observed ECG changes with PNS on CAD patients. Positive correlations of PNS and improvements of ECG were reported that ischaemic changes on ECG were attenuated significantly. A RCT divided 100 patients randomly into treatment group and control group. The two groups were given conventional drugs, treatment group plus PNS for 4 weeks. It's elucidated that ECG in the treatment group were better than those in the control group, in company with the curative effect of angina pectoris, FAA, the rate of stopping and the dosage of nitroglycerin (Song et al., 2005). Another RCT reported that with PNS treatment, ECG of $92 \%$ CAD patients returned to normal state or rise more than $0.05 \mathrm{Mv}$ of ST segment depression, different significantly with conventional drugs alone (Hou, 2016).

\section{Lipid Metabolism}

Lipid disorder is one of the main risk factors for CAD. A $20 \%$ reduction in major coronary events within 5 years was caused by a decrease of $1 \mathrm{mmol} / \mathrm{L}$ in LDL level (Baigent et al., 2005). All three RCTs reported effectiveness of PNS on lipids of CAD patients. PNS could decrease significantly TC, TG, LDL. PNS combined with conventional drugs was more effective than conventional drugs alone. In addition, some researches 
were trying to evaluate PNS alone with atorvastatin. Seventyone patients with CAD were randomly divided into two groups: PNS group (36 cases) and atorvastatin group $(n=35)$. PNS was given $100 \mathrm{mg}$ orally, while atorvastatin group received atorvastatin $20 \mathrm{mg}$ orally. The results showed that there was no significant difference in the levels of TG, TC, CIMT, and plaque between two groups before and after treatment. There was no significant difference in LDL-C before and after treatment in PNS group, while the LDL-C descended significantly in atorvastatin group. The incidence of abnormal liver function, gastrointestinal reaction, and recurrent cardiovascular events in patients with atorvastatin was significantly higher than PNS (Feng et al., 2016).

In this overview, 15 RCTs observe the effect of PNS as alternative and complementary medicine on secondary outcomes, such as frequency of angina attack, duration of angina attack, ECG and lipid metabolism. And the results illustrated PNS combined with conventional drugs had also significant effects on changing the secondary outcomes.

\section{ADVERSE EVENTS}

A systematic review evaluated the safety of PNS for UA, including six RCTs with 716 participants. Four of the included trials $(66.7 \%)$ reported adverse effects related to treatment with PNS combined with conventional drugs. The only reported adverse effect was rash at $0.27 \%(1 / 363)$. No severe adverse events were reported (Yang et al., 2013). Another systematic review evaluated an oral $P$. notoginseng preparation for CAD and included 17 randomized clinical trials with 1,747 participants. Nine trials reported adverse events. One trial reported reduced blood pressure and increased heart rates. One trial reported nausea, dizziness, and vomiting. One trial reported erythra, and six trials indicated no adverse events throughout the duration of treatment (Shang et al., 2013).

Focusing on PNS for CAD, nine RCTs reported adverse events in all 17 RCTs. No observable toxicity in liver or kidney function was measured by serum markers. Several RCTs described adverse events that indicated that oral PNS for CAD is not related to adverse reactions (Table 4). Feng et al. (2016) reported 2 cases with elevated transaminase, 1 case with muscle pain, and 1 case with gastrointestinal discomfort in the control group. No obvious adverse reactions were observed in the treatment group (Feng et al., 2016). In the experimental group of Yan et al. (2015), 1 case of subcutaneous hemorrhage and 1 case of positive fecal occult blood occurred. One case of nausea and 1 case of positive fecal occult blood occurred in the control group (Yan et al., 2015). In $\mathrm{Yu} 2010,1$ case in the experimental group showed a small amount of rash after $3 \mathrm{~d}$ of treatment, which was not caused by the treatment $(\mathrm{Yu}, 2010)$. No significant difference was observed in the incidence of adverse reactions. Furthermore, PNS was not related to any obvious abnormalities in liver and kidney function.

\section{THE MECHANISMS OF PNS ON CAD}

CAD occurs when atherosclerotic lesions impede blood flow in the coronary artery. The plaque activation causes ischaemia and infarction. Ruptures tend to happen near the thin and easy destroyed fibrous cap where activated immune cells, inflammatory molecules, and proteolytic enzymes are abundant (Santos-Gallego et al., 2014). They can weaken the cap and transform stable plaque to an unstable vulnerable plaque that is more likely to rupture. The pathomechanism of atherosclerosis in CAD is related to inflammation, lipid metabolism, endothelial erosion, coagulation system dysfunction, and apoptosis (Hopkins, 2013). Plaque rupture is the major trigger of CAD (Hansson, 2005), while hypoxia and ischaemia are the pathological manifestations of the disease. In addition, angiogenesis can improve blood flow in the presence of microvascular blockage in CAD.

Saponins are a group of natural compounds in plants and foods. PNS is the most important compound among $P$. notoginseng's effective components. In the past 10 years, it had received extensive attention in the treatment of CAD at home and abroad. Many studies showed that it had anti-inflammatory, antiapoptotic, anti-hypoxic, lowering lipids, anti-coagulation, and pro-angiogenesis properties (Table 5, Figure 4).

\section{Anti-inflammation}

Inflammation dominates in CAD and atherosclerosis. Immune cells gather in the early atherosclerotic lesions, where effector molecules promote the progress of inflammation which can induce acute coronary syndrome (ACS; Han, 2008). In vulnerable plaques, the main characteristic is that inflammation exists widespread. Several studies showed that different systems of inflammation markers such as C-reactive protein in patients are

TABLE 4 | The incidence of adverse reactions with PNS for CAD.

\begin{tabular}{|c|c|c|c|}
\hline Adverse events & $\begin{array}{l}\text { The incidence of adverse } \\
\text { reactions (experimental) }\end{array}$ & $\begin{array}{l}\text { The incidence of adverse } \\
\text { reactions (control) }\end{array}$ & References \\
\hline Elevated transaminase & $0 / 36$ & $2 / 35$ & Feng et al., 2016 \\
\hline Gastrointestinal discomfort & $0 / 36$ & $1 / 35$ & Feng et al., 2016 \\
\hline Muscle pain & $0 / 36$ & $1 / 35$ & Feng et al., 2016 \\
\hline Subcutaneous hemorrhage & $1 / 28$ & $0 / 27$ & Yan et al., 2015 \\
\hline Fecal occult blood positive & $1 / 28$ & $1 / 27$ & Yan et al., 2015 \\
\hline Nausea & $0 / 28$ & $1 / 27$ & Yan et al., 2015 \\
\hline Rash & $1 / 50$ & $0 / 50$ & Yu, 2010 \\
\hline Total & $3 / 214$ & $6 / 208$ & \\
\hline
\end{tabular}




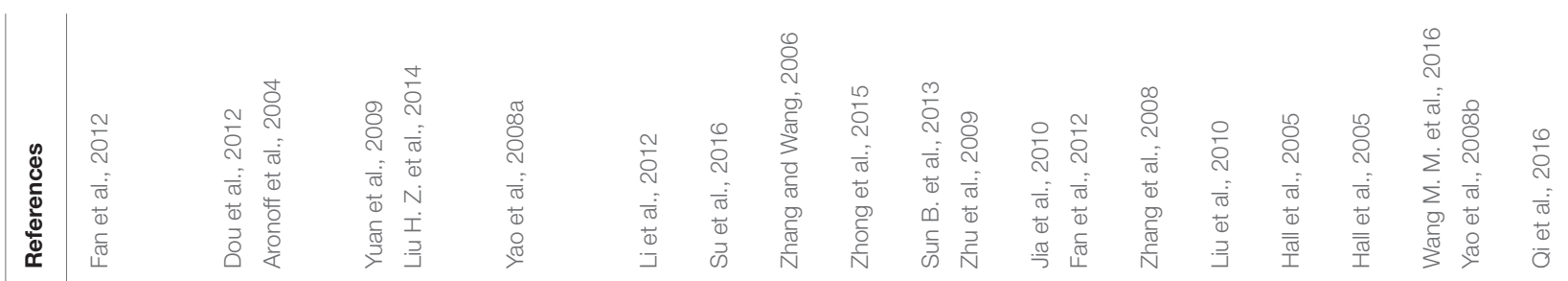
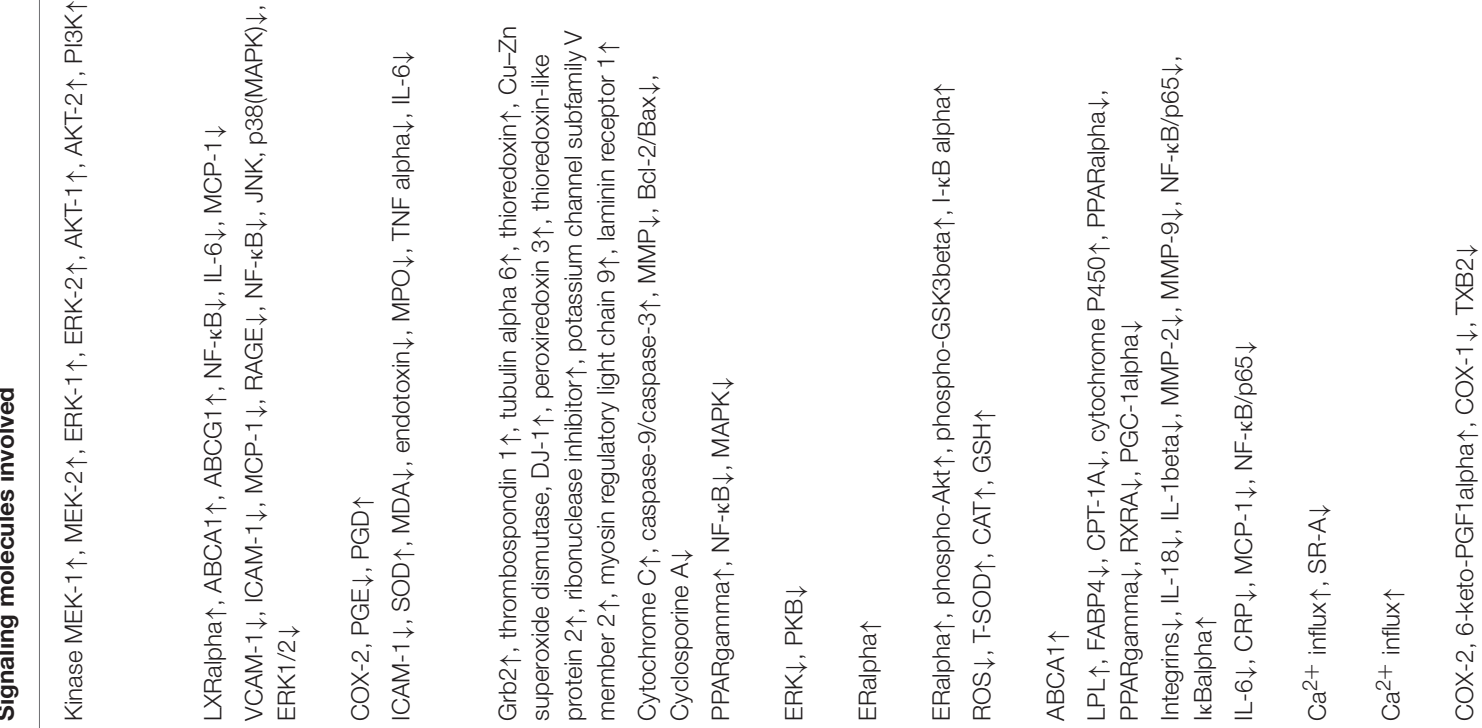

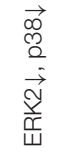
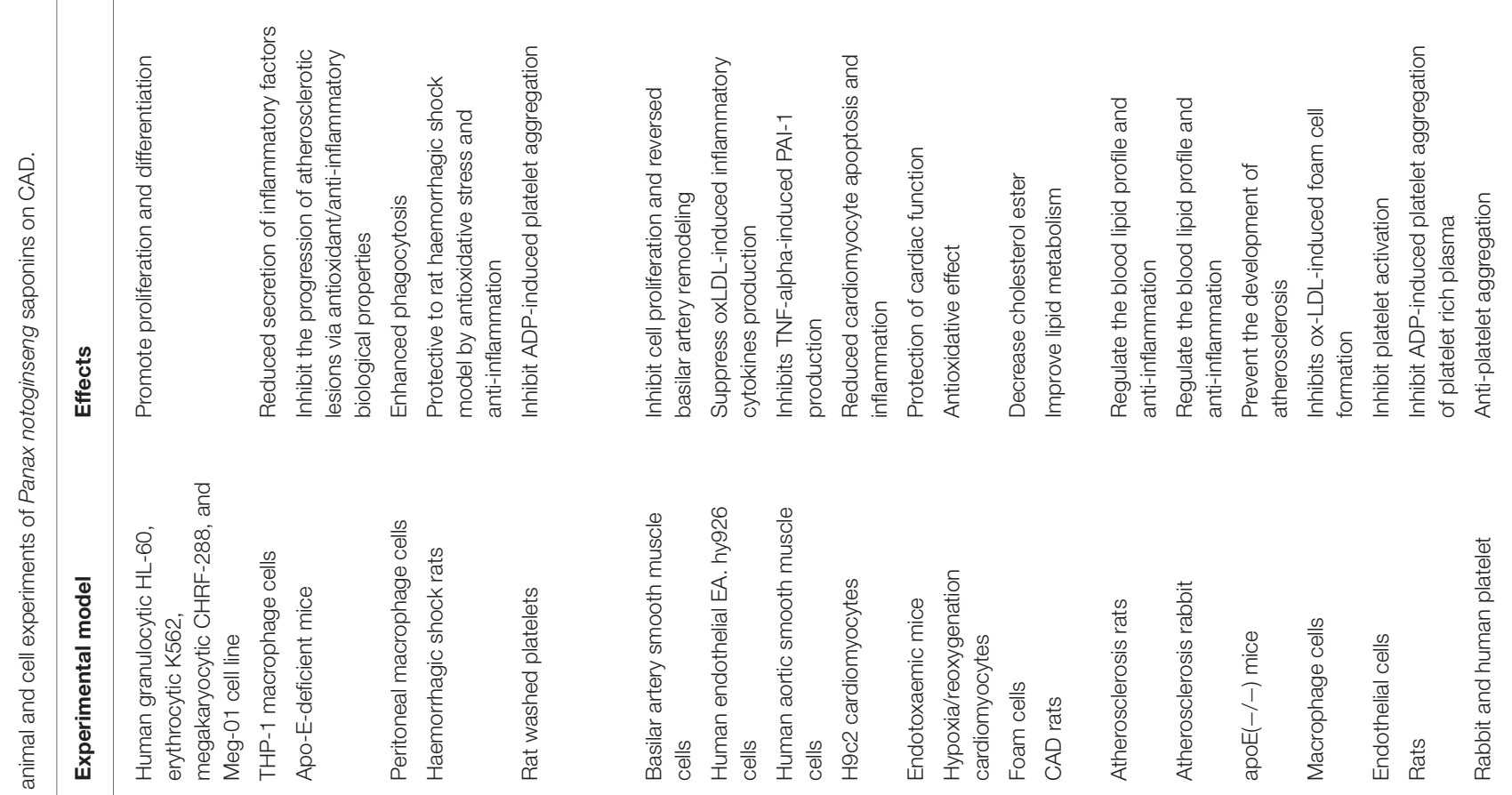

高旁员

올

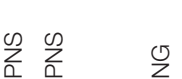

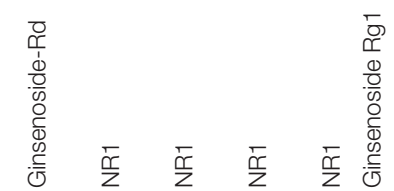

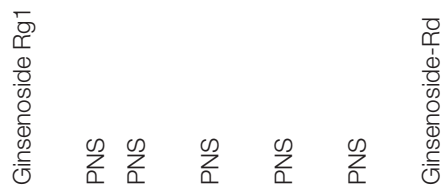

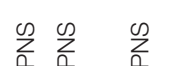




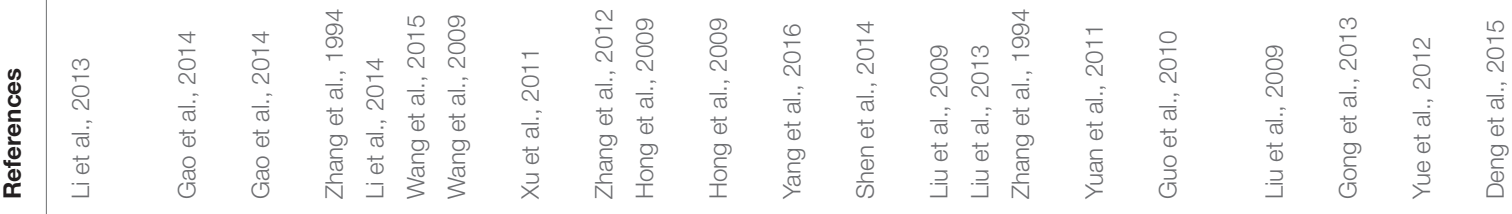

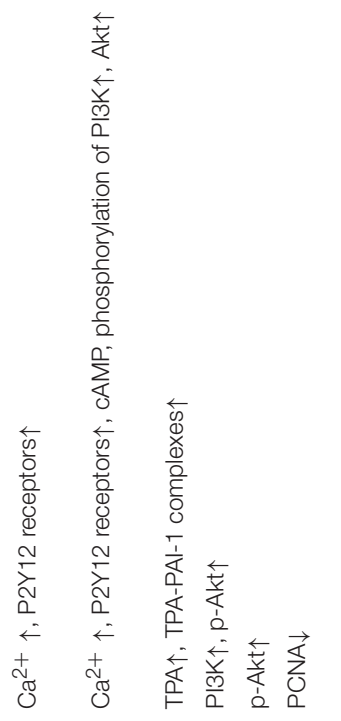

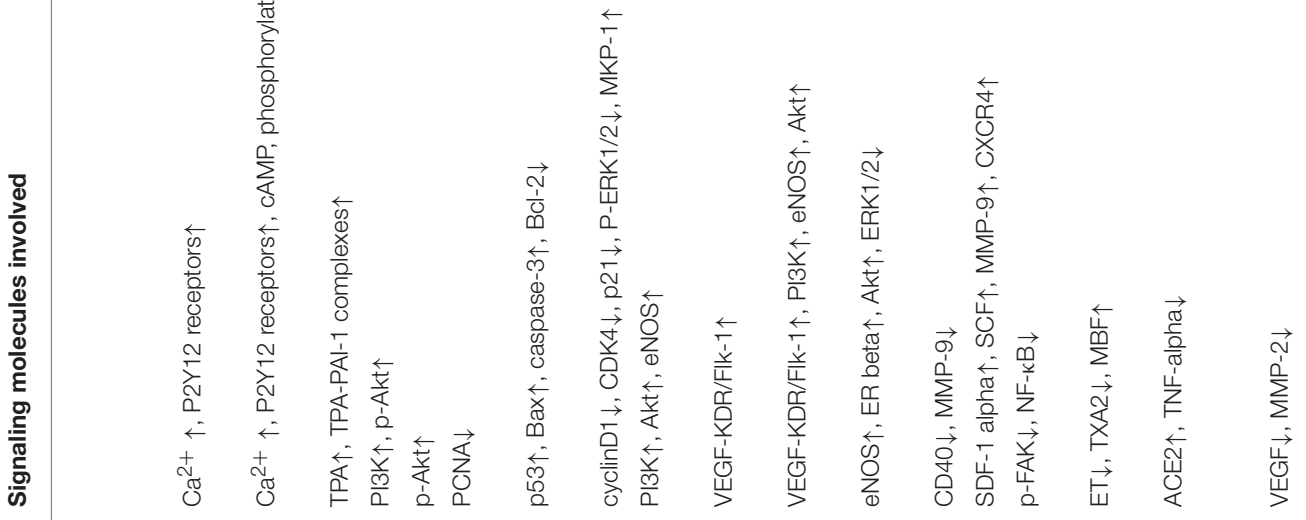

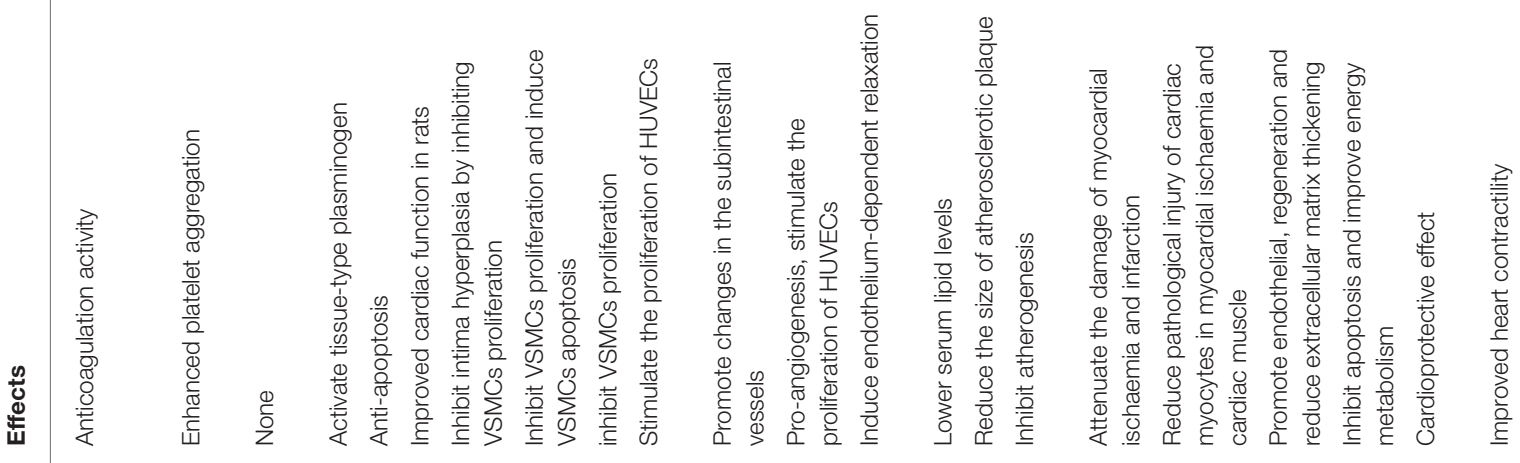

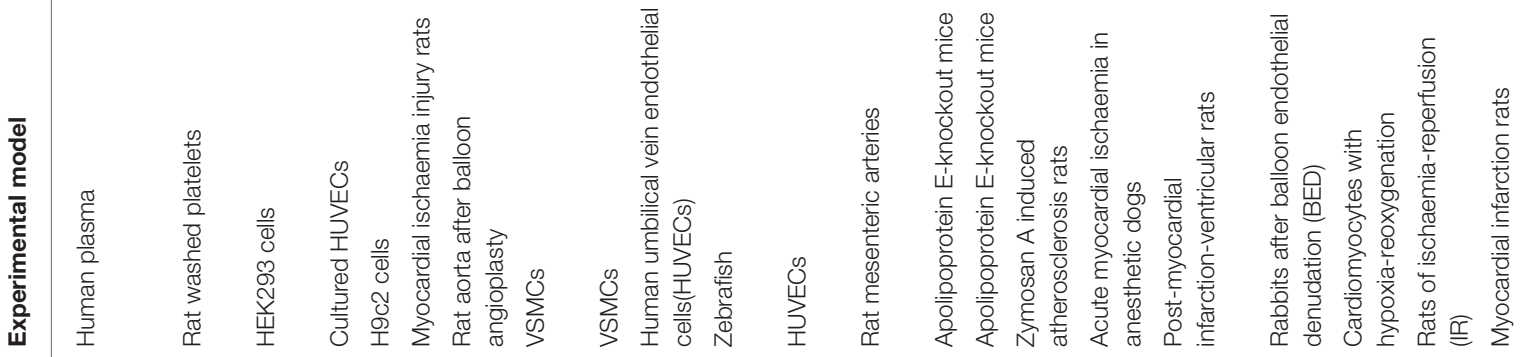

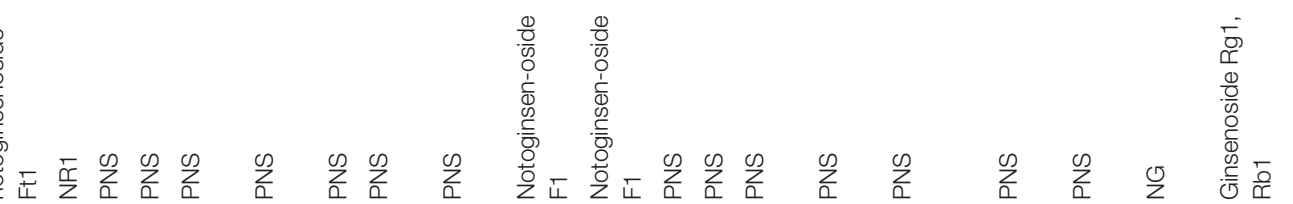




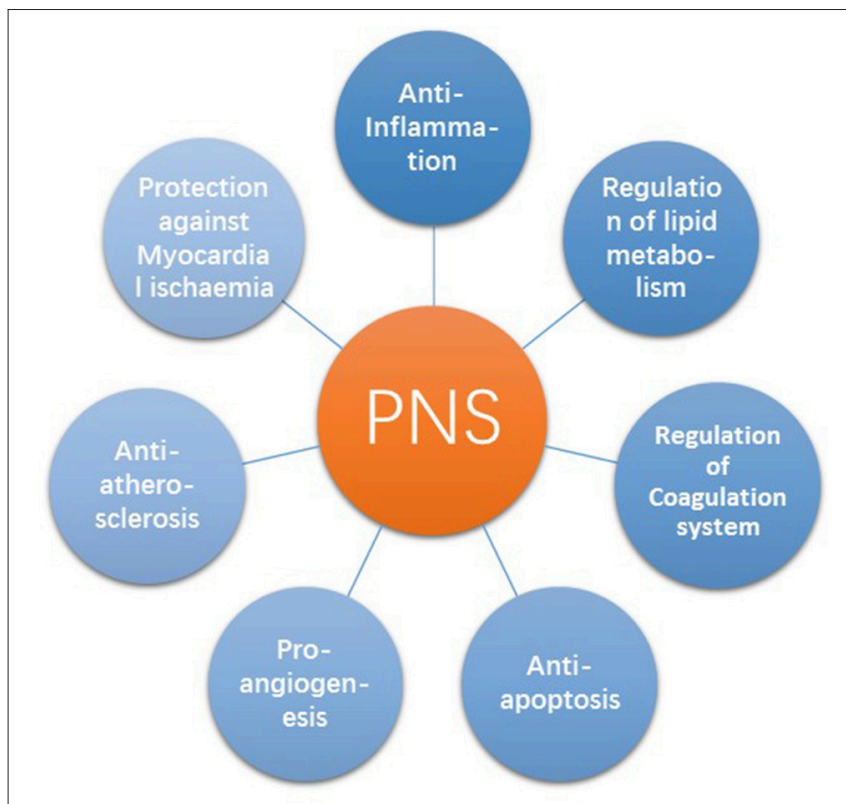

FIGURE 4 | Summary of seven main functions of PNS in CAD.

related to an increased risk of ACS (Crea and Liuzzo, 2013; Mann et al., 2014). And inflammation associated with CAD includes MAPK activation, the role of NF- $\kappa \mathrm{B}, \mathrm{TNF}-\alpha$, ROS for signaling, modified lipoprotein on endothelial cells and other cell activation, leukocyte adhesion, formation of foam cells, macrophages (Figure 5A), and vascular smooth muscle cells (VSMCs).

First, NF- $\kappa \mathrm{B}$ is critical for the trigger and development of atherosclerosis (Hopkins, 2013). The elevated NF-кB activators such as osteoprotegerin were related to increased mortality, especially of cardiovascular diseases (Venuraju et al., 2010). Activation of NF- $\kappa$ B led to regulation of many atherogenic genes, facilitation of inflammation, induction of chemokines, and adhesion molecules on the surface of endothelial cell (ECs) (Figure 5B). Chemokines and adhesion molecules attracted the monocytes to the location prone to atheroma (Gerits et al., 2007; Marks et al., 2009; Baker et al., 2011). PNS inhibited NF$\kappa \mathrm{B}$ DNA binding activity (Sun X. et al., 2013) and secreting pro-inflammatory factors, interleukin (IL)-6 and MCP-1 in macrophages (Fan et al., 2012). The size of atherosclerotic lesions and the number of macrophages in apolipoprotein $\mathrm{E}$ (apoE; $-/-)$ mice were reduced by PNS. In addition, PNS reduced the expression of proinflammatory cytokines VCAM-1, ICAM1 , and MCP-1 with inhibition of NF- $\mathrm{B}$, JNK, p38 (MAPK), and ERK1/2 activation and RAGE (Dou et al., 2012). Phagocytosis induced the expression of the pro-inflammatory factor COX2 and the production by COX-2 regulated the functions of macrophage (Aronoff et al., 2004). In the model of inflammation, the expression of COX-2 reached a peak. However, in the later stage COX-2 descended which leads to activation of PPAR $\gamma$ and inhibition of inflammation by NF- $\kappa$ B. Interestingly, PNS suppressed the expression of COX-2 at an early stage with promotion of phagocytosis, while PNS also elevated COX-2 expression at a later stage (Yuan et al., 2009).

Second, oxidation is generally considered as a facilitator or a modulator of inflammatory signaling (OliveiraMarques et al., 2009), and also a major endothelial-derived hyperpolarizing factor (EDHF) mediator (Capettini et al., 2010). Oxidative stress involves the inflammation of vessels and the progression of atherosclerosis. Excessive reactive oxygen species (ROS) generation has been suggested to up-regulate pro-inflammatory cytokines and adhesion molecules which can result in atherosclerosis initiation consequently (Zhang et al., 2009). ROS facilitated the activation of ROS/Akt/IKK pathways that interact with NF- $\mathrm{B}$ (Qi et al., 2012). In addition, MDA(malondialdehyde) is a product of a free-radical peroxidatic reaction on lipids, and Superoxide dismutase (SOD) is a free-radical scavenger (Van Raamsdonk and Hekimi, 2012). Myeloperoxidase (MPO), a peroxidase enzyme, could accurately predicted the mortality risk in patients with coronary angiography. The improvement of MPO and CRP ameliorated the long-term risk assessment of outcomes in CAD patients (Heslop et al., 2010).

PNS also are considered as free radical-scavengers with antioxidant properties. PNS could impede the development of atherosclerotic lesions through the antioxidant and antiinflammatory effects (Aronoff et al., 2004). PNS protected a rat haemorrhagic shock model via antioxidative stress and anti-inflammatory pathways. PNS also increased SOD activity, decrease MDA, endotoxin, MPO, TNF alpha, and IL-6 (Liu H. Z. et al., 2014). PNS could reduce oxidative stress and inhibit plaque progression. SOD and glutathione activities were elevated and ROS generation is impaired in apoE $(-/-)$ mice treated with PNS (Aronoff et al., 2004).

Treatment with Notoginsengnosides (NG) could decrease the ROS level in platelets (Shang et al., 2013). GinsenosideRd significantly promoted $\mathrm{H}_{2} \mathrm{O}_{2}$-induced cell apoptosis with a concentration-dependent manner (Li et al., 2012). NR1 with the effect of phytoestrogen, was illustrated as a component with anti-inflammatory, antioxidative and anti-apoptotic properties. NR1 can restrict oxidized low-density lipoprotein (ox-LDL)induced inflammatory cytokines including NF- $\kappa$ B, MAPK, TNFalpha, and IL-1 beta (Su et al., 2016). It also inhibits PAI1 overexpression by TNF-alpha in human aortic smooth muscle cells (HASMCs) and the ERK/PKB pathways (Zhang and Wang, 2006). NR1 can protect the heart from septic shock, probably through the activation of ER alpha and PI3K/Akt pathway. This mechanism blocked NF- $\kappa$ B activation and attenuated inflammation and apoptosis in the myocardium (Sun B. et al., 2013; Zhong et al., 2015). In addition, the pretreatment with ginsenoside $\mathrm{Rg} 1$ decreased the release of lactate dehydrogenase(LDH) and increased cell viability dosedependently. Ginsenoside Rg1 suppressed ROS and $\mathrm{Ca}^{2+}$ level intracellularly by raising the activity of endogenous antioxidants as T-SOD, CAT, GSH (Zhu et al., 2009).

\section{Regulation of Lipid Metabolism}

Lipoprotein disorder is one of the main risk factors of CAD. A meta-analysis of 14 randomized trials showed that a decrease of 


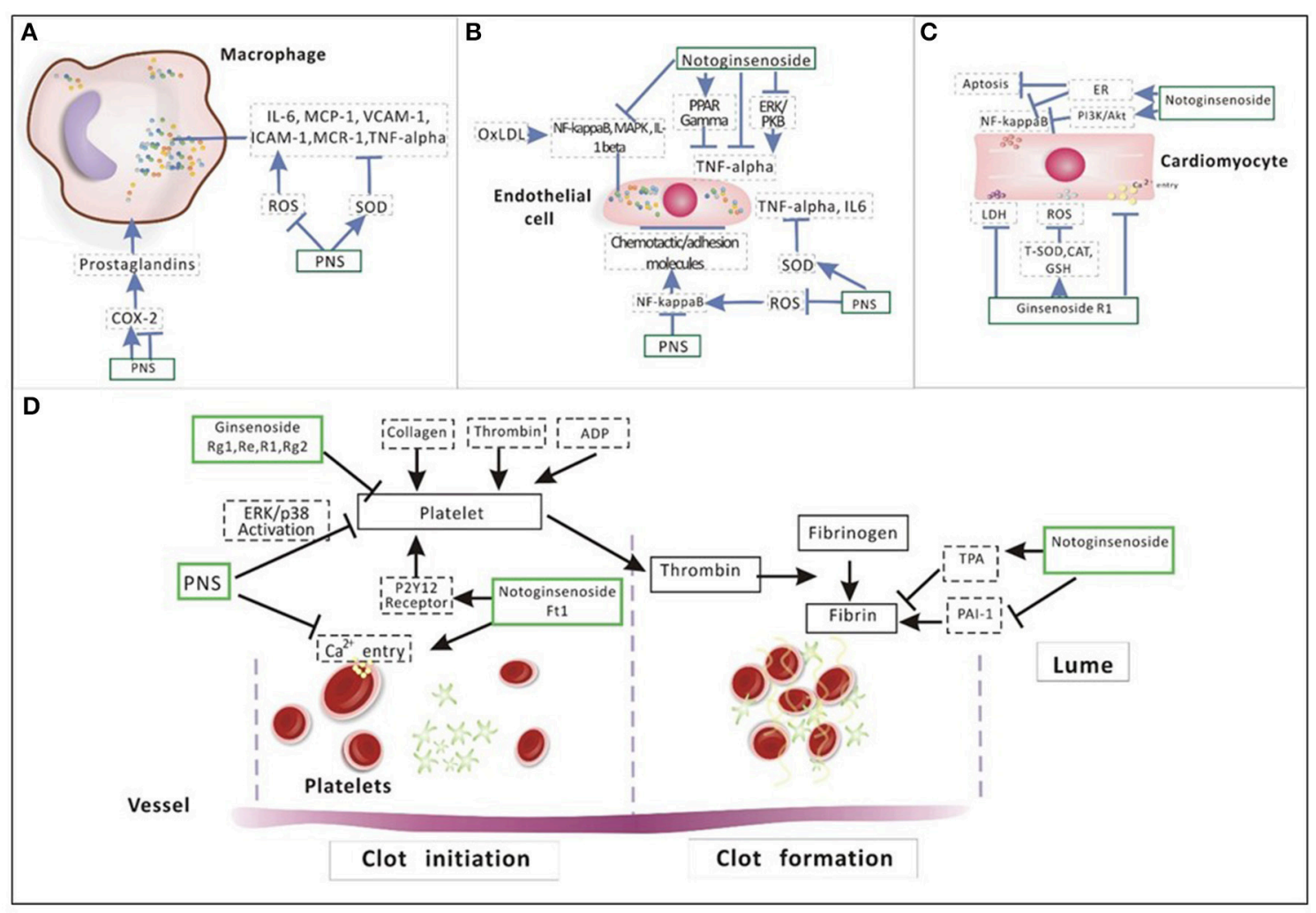

FIGURE 5 | Illustration of the mechanism of PNS on (A) macrophage, (B) endothelial cell, (C) cardiomyocyte, and (D) platelet aggregation. In the process of inflammation among macrophages (A), pro-inflammatory factors such as IL-6, MCP-1, VCAM-1, ICAM-1, MCR-1, and TNF-alpha are regulated by ROS and SOD. Prostaglandins produced by COX-2 is negatively related to phagocytosis. PNS can regulate pro-inflammatory factors by inhibiting ROS and promoting SOD. In addition, PNS also inhibit TNA alpha directly. The activated NF-kB regulates the expression of many atherogenic genes, creating a local inflammatory condition and inducing chemotactic factors and adhesion molecules on the surface of ECS (B). PNS can increase SOD activity by decreasing TNF alpha, IL-6 and ROS generation. Notoginsenoside R1 can suppress inflammatory cytokines production by activating PPAR gamma and by suppressing ERK and PKB, inhibiting TNF-alpha. In addition, NR1 can inhibit NF-KB, MAPK, IL-1 beta and reduce cardiomyocyte apoptosis and inflammation through the activation of ER alpha and PI3K/Akt signaling (C). Ginsenoside Rg1 reduced intracellular ROS and LDH and suppressed the intracellular Ca2+ level by increasing the activity of endogenous antioxidants, including T-SOD, CAT and GSH. About (D), NG have an inhibitory effect on platelet aggregation. The effect of PNS in anti-platelet aggregation is related to the suppression of intracellular calcium mobilization and ERK2/p38 activation. Three main ginsenosides (Rg1, Re, and R1) that exist in PNS also showed anti-platelet activity. Ft1 induced dose-dependent platelet aggregation mediated through P2Y12 receptors. NR1 significantly decreased TNF alpha-induced PAl-1.

$1 \mathrm{mmol} / \mathrm{L}$ in plasma LDL levels generates a $20 \%$ reduction in major coronary events including coronary revascularization and stroke within 5 years (Baigent et al., 2005). CAD is closely related to lipid metabolic disorders, specifically increased triglycerides (TG), low-density lipoprotein cholesterol (LDL-C), ox-LDL, and total cholesterol (TC; Labreuche et al., 2010; Nyyssonen et al., 2012). PNS can reduce remarkably the level of cholesterol ester in foam cells by up-regulation of ABCA1. This bioactivity may be related to the special chemical structures of PNS that are like the natural agonist of liver $\mathrm{X}$ receptor alpha (LXR alpha; Jia et al., 2010). LXR $\alpha$ as a key regulator of macrophage function, controls transcriptional programmes involved in lipid metabolism and inflammation (Christoffolete et al., 2010). PNS could regulate lipids by activation of the LXR alpha gene promoter which increased $A B C A 1$ and ABCG1 subsequently and suppressed NFкB DNA binding activity (Fan et al., 2012).

PNS could markedly reduce TC, TG, and LDL-C (Zhang et al., 2008) and increase high-density lipoprotein cholesterol (HDL-C) significantly (Liu et al., 2010). CPT-1A is a key enzyme in the process of fatty acid oxidation. While the fatty acid transporter protein 4 (FATP4) is associated with long and very long chain fatty acid uptake and promoting synthesis of acyl-CoA (Hall et al., 2005). Fatty acid binding protein 4 (FABP4) and CPT-1A, were downregulated in ischemic zone of the heart. PNS could regulate lipid metabolism by increasing the expression of FABP4 and CPT-1A (Wang Q. et al., 2016).

Lipid metabolic disorder can be caused by inflammation and can exasperate the inflammation (Hotamisligil, 2006). Dyslipidemia and inflammation accelerate each other to form a detrimental cycle. Regulation of lipid metabolism disorders is conducive to inflammation alleviation and anti-inflammatory effects benefits the maintenance of balanced lipid metabolism. PNS could regulate lipid metabolism. Meanwhile, PNS decreased significantly the expressions of some inflammatory cytokines including integrins, IL-18, IL-1 beta, and matrix metalloproteinases 2 (MMP2) and 9 (Zhang et al., 2008). 
In conclusion, $\mathrm{CAD}$ is closely related to lipid metabolic disorders, specifically including increased TG, LDL-C, ox-LDL, and TC. PNS could depress the level of TC by elevating LXR alpha, ABCA1, and ABCG1 and reducing NF-кB. In addition, PNS can regulate lipid metabolism by inhibiting LPL and increasing FABP4 and CPT-1A. Furthermore, lipidosis is closely related to inflammation, which PNS have diverse effects on.

\section{Regulation of Coagulation System}

In CAD, antiplatelet therapy has become an important treatment according to several important guidelines (Chew et al., 2016; Levine et al., 2016). Near wound, platelets are recruited to restore endothelial integrity to initiate thrombus formation (Nording et al., 2015). Thrombosis is associated with platelet aggregation. Thromboxane A2 (TXA2), derived from platelets, induces powerfully release and aggregation of platelets.

PNS inhibited platelet activation by multiple ingredients and pathways. PNS could decrease platelet activation, inhibit adhesion and aggregation of platelet, prevent thrombosis, and improve microcirculation (Wang et al., 2004). PNS protected ECs from injury by suppressing platelet adhesion, in which PNS was superior to aspirin. The underlying mechanism is related to the COX pathway in both ECs and platelets (Figures 5B,D; Wang M. M. et al., 2016). NG could suppress platelet aggregation in vitro. Furthermore, in vivo NG could also significantly inhibit platelet aggregation of platelet rich plasma (PRP; Yao et al., 2008b). The effect of PNS in anti-platelet aggregation is associated with inhibition of intracellular calcium mobilization and activation of ERK2/p38. Three main ginsenosides (Rg1, Re, and NR1) existing in PNS also demonstrated antiplatelet activity, but their combination did not exhibit any synergistic effect on rabbit platelet aggregation (Qi et al., 2016). Rg1 and $\operatorname{Rg} 2$ can significantly prolong the clotting time. Compared with Rg1, Rg2 showed a stronger anticoagulant effect (Li et al., 2013).

However, in PNS, notoginsenoside Ft1 as the potent procoagulant component induced platelet aggregation dosedependently. The P2Y12 receptor serves as a crucial regulator of haemostasis and thrombosis on the platelet. When conditioned by $\mathrm{ADP}$, the $\mathrm{P} 2 \mathrm{Y} 12$ receptor activated a series of downstream events that result in platelet aggregation, shape change, dense granule secretion (Dorsam and Kunapuli, 2004; Gao et al., 2014). Ft1 deceased plasma coagulation indexes and tail bleeding time and increased thrombogenesis and cytosolic $\mathrm{Ca}^{2+}$ accumulation.

Fibrinolysis is part of the coagulation cascade, which is adjusted by plasminogen activator (PA) and PA inhibitor (PAI-1). Abnormal fibrinolysis and high plasma concentrations of PAI1 are related to an increased risk of CAD (McBane et al., 2010). When human umbilical vein endothelial cells (HUVECs) were conditioned with purified NR1, tissue-type PA (TPA) synthesis increase in a dose- $(0.01-100 \mathrm{mg}$ of NR1/mL) and time-dependent manner. NR1 significantly decreased PAI-1 mRNA, protein and secretion in HASMCs in a dose-dependent manner (Zhang and Wang, 2006). TPA activity and TPA-PAI-1 complexes reached greater than two-fold and threefold maximal stimulation, respectively NR1. In contrast, NR1 induced five-fold decrease in PAI-1 activity (Zhang et al., 1994).

\section{Anti-apoptosis}

Myocardial ischaemia can lead to widespread cell apoptosis (Ohno et al., 1998). PI3K/Akt pathway is an important regulator including proliferation, apoptosis and nitric oxide (NO) synthesis (Blanes et al., 2007). PI3K also strengthens the oxidative capacity of cardiac fatty acid. The PI3K signaling cascade diminishes myocardial damage by ischaemia via recruiting several endogenous cardioprotective pathways (Hausenloy et al., 2005).

PNS could protect myocardial cells from apoptosis induced by ischaemia both in vitro and in vivo by activating the PI3K/Akt signaling pathway (Tello-Montoliu et al., 2006). PNS significantly up-regulated p-Akt in $\mathrm{H} 9 \mathrm{c} 2$ cells and ischaemic myocardial tissues. PNS attenuated cell apoptosis via chromatin concentration and condensation by up-regulating the antioxidative abilities of SOD and MDA (Li et al., 2014). PNS also improved cardiac function in the left ventricular ejection fractions (EF) of rats (Chen et al., 2011; Wang et al., 2015).

The pathological proliferation of VSMCs is a crucial factor involved in the pathogenesis of atherosclerosis, associated with inflammation, apoptosis, and matrix alterations (Zakar and Ken, 2003). PNS suppressed proliferation and induced apoptosis in VSMCs (Wang et al., 2009) by up-regulating p53, Bax, and caspase-3 and down-regulating Bcl-2 (Xu et al., 2011). In addition, both atorvastatin and PNS have been observed to suppress VSMC proliferation by inhibiting the activation of the ERK signaling pathway (Zhang et al., 2012).

\section{Pro-angiogenesis}

Angiogenesis is the stimulation of the endothelium to shape new blood vessels, which is implicated in the pathophysiology of CAD (Tello-Montoliu et al., 2006). In CAD, inflammation related to atherogenesis contributes to the interaction of angiogenic factors (Patel et al., 2006), which lead to vascular repair (Chong et al., 2004). Various angiogenic growth factors and progenitor cells can promote the formation of new blood vessels (Mitsos et al., 2012). Angiogenesis is a potential treatment in many physiological processes such as MI, chronic cardiac ischaemia, and stroke (Giacca and Zacchigna, 2012).

PNS could enhance angiogenesis and the proangiogenic effects including the VEGF-KDR/Flk-1 and PI3K-Akt-eNOS signaling pathways in vivo and in vitro (Hong et al., 2009). NR1, similar to $\operatorname{Rg} 1$ and Re, had been shown to have pro-angiogenic effect, possibly by activation of the VEGF-KDR/Flk-1 and PI3KAkt-eNOS signaling pathways in vivo and in vitro (Yang et al., 2016).

Ft1 can stimulate angiogenesis. Ft1 led to proliferation, migration and tube formation in HUVECs by activation of the PI3K/Akt and ERK1/2 pathways in rat mesenteric arteries. This leads to the phosphorylation of eNOS and release of NO, which triggers soluble guanylyl cyclase in the VSMCs (Shen et al., 2014).

\section{Anti-atherosclerosis}

Atherosclerosis is the pathological basis of CAD. Furthermore, the development of chronic atherosclerosis to form thrombosis is the pathogenesis of ACS (Mann et al., 2014). ApoE is a ligand for cleansing receptors of chylomicrons and very low 
density lipoprotein residues. The lack of apoE can lead to the accumulation of cholesterol-rich residues in plasma, and long-time accumulation can generate atherosclerosis (Heeren et al., 2006) with hypercholesterolemia and spontaneous arterial lesions (Meir and Leitersdorf, 2004). PNS was able to decrease lipids, ox-LDL in serum and the expressions of CD40 and MMP-9 in apoE(-/-) mice (Liu et al., 2009). Meanwhile, PNS lessened the size of atherosclerotic plaques, partly by progenitor cell mobilization. PNS also augmented endothelialization and reduced the VSMC content of the lesions (Liu et al., 2013).

A high-fat diet together with Zymosan (Zym) induces atherogenesis in rats. PNS reduced the levels of TC, TG, LDLC, IL-6, and C-reactive protein and increased the HDL-C level significantly in serum of atherosclerosis rabbits by inhibiting FAK phosphorylation, integrins expression and NF- $\mathrm{B}$ translocation (Yuan et al., 2011). And PNS significantly down-regulated MCP1 and NF-кB/p65 with 8 weeks of treatment (Liu et al., 2010).

Ginsenoside Rd, isolated from PNS, is a voltage dependent $\mathrm{Ca}^{2+}$ channel blocker. Ginsenoside Rd decreased remarkably the size of atherosclerotic plaque and ox-LDL of macrophage in the apoE $(-/-)$ rats. In vitro, ginsenoside $\mathrm{Rd}$ suppressed the formation of foam cells induced by ox-LDL and cholesterol accumulation in macrophages (Li et al., 2011).

\section{Protection against Myocardial Ischaemia}

PNS exerted a certain degree of improvement on myocardial ischaemia (Tang et al., 2010). In fact, the earliest study to demonstrate the effects of $P$. notoginseng on CAD was a study published in 1972, in which the oral administration of $P$. notoginseng reduced the dosage requirement of nitroglycerin and improved ECG in clinic. And P. notoginseng significantly increased coronary blood flow in dogs (Department of Cardiology in Second Affiliated Hospital of Wuhan Medical College, 1972). PNS was shown to obviously alleviate the degree of myocardial ischaemia and narrow the ischaemic area subjected to myocardial ischaemia and infarction (Fu et al., 2006). PNS could enhance left ventricular systolic and diastolic functions, decrease peripheral resistance, and improve the cardiac function of rats with post-myocardial infarction left ventricular remodeling (Guo et al., 2009). The endothelium was denudated completely after balloon endothelial denudation (BED). PNS could sustaine anti-restenotic effects after BED injury. PNS promoted endothelial regeneration and reduced ECM thickening (Chen et al., 2004). In vitro, PNS exhibited an anti-apoptotic effect both in oxygen-deprived H9c2 cells and in ischaemic myocardial tissues (Chen et al., 2011). In addition, PNS could decrease the pathological injury to cardiac myocytes with ischaemia and improve ventricular remodeling (Guo et al., 2010).

The PPAR family, a series of transcription factors, regulates cardiac energy metabolism and impacts metabolism of cardiac fatty acid and glucose (Madrazo and Kelly, 2008). PGC- $1 \alpha$ is a transcriptional coactivator of the PPARs and a critical factor in myocardial metabolism (Huss et al., 2004). In ischaemic rats, transcriptional factors were downregulated such as PPARs, RXRA, and PGC-1 alpha (Wang Q. et al., 2016). PNS could up-regulate expressions of these factors.

In addition, salvianolic acids' compatibility with PNS could protect cardiomyocytes (Figure 5C) during hypoxia and reoxygenation injury by inhibiting apoptosis and improving energy metabolism compared to any single drug (Gong et al., 2013). In the model of ischaemia/reperfusion, salvianolic acids (SA), NG, and a combination of SA and NG exhibited the cardioprotective effects. SA and NG displayed both similarities and differences in pathways such as energy metabolism, lipid metabolism, cell proliferation, and apoptosis (Yue et al., 2012). The combination of SalB and Rg1, instead of SalB and Rb1, advanced cardiac contractility in rats with MI (Deng et al., 2015).

\section{DISCUSSION}

\section{Summary of Current Evidence}

In the past two decades, a breakthrough has been achieved in the pharmacology of PNS. The knowledge of PNS functions offers a new opportunity for the prevention and treatment of CAD. PNS has been observed to have multiple positive effects in the key processes of $\mathrm{CAD}$, including anti-inflammation, the regulation of lipid metabolism and the coagulation system, anti-apoptosis, pro-angiogenesis, anti-atherosclerosis, and anti-myocardial ischaemia (Figure 6). Several RCTs have shown that the long-term use of PNS can effectively reduce the end-point of CAD (Meng et al., 2013; Yan et al., 2015). In addition, many RCTs found that PNS can also significantly improve performance on the ECG and reduce the frequency and the duration of angina attacks, greatly regulating the lipids (Song et al., 2005; Han, 2008; Liu et al., 2008; Du and Chen, 2009; Zhou and Bai, 2009; Wei, 2010; Yu, 2010; Kuang et al., 2011; Wan, 2011; Meng et al., 2013; Teng, 2014; Zhang, 2014; Zheng, 2014; Yan et al., 2015; Hou, 2016).

The function of PNS on platelet aggregation resembles aspirin. For the patients with aspirin resistance (Arachidonic acid inhibitory rate $<50 \%$ ) and clopidogrel resistance (Adenosine diphosphate inhibition rate $<30 \%$ ), Ticagreloran, an oral reversibly binding P2Y12 inhibitor, is commonly used alternative drug (Nylander and Schulz, 2016). However, Ticagrelor is unsuitable for patients with bleeding tendency, meanwhile it means a big financial burden on patients or the government. For those patients, PNS is recommend for anti-platelet aggregation. Several studies in vitro and in vivo PNS may inhibit the activation of platelet through multiple components and multiple pathways. In a RCT, PNS alone is illustrated to decrease platelet aggregation and less adverse events (Yu, 2010).

Statins as a main drug by many guidelines have shown good effects in the primary and secondary prevention of CAD (Harris et al., 2015; Jellinger et al., 2017). However, statins have side effects including elevated liver transaminase, myopathy, myalgia, myositis, and even rhabdomyolysis. PNS could regulate lipid metabolism, not so strongly as statins, but with a higher safety. Meanwhile PNS has the roles of anti-inflammation and antiplatelet aggregation. So, for patients with liver damage, or the elderly without high TC, TG, LDL, we recommend PNS for lipid-lowering and anti-inflammation.

In addition, nitroglycerin is one of the oldest of cardiovascular drugs in clinics. Nitroglycerin exerts anti-ischemic effect mainly by expanding capacity vein, reducing preload and releasing coronary artery spasm. PNS could reduce myocardial ischemia and relieve angina pectoris. In clinic PNS has similar effects and 


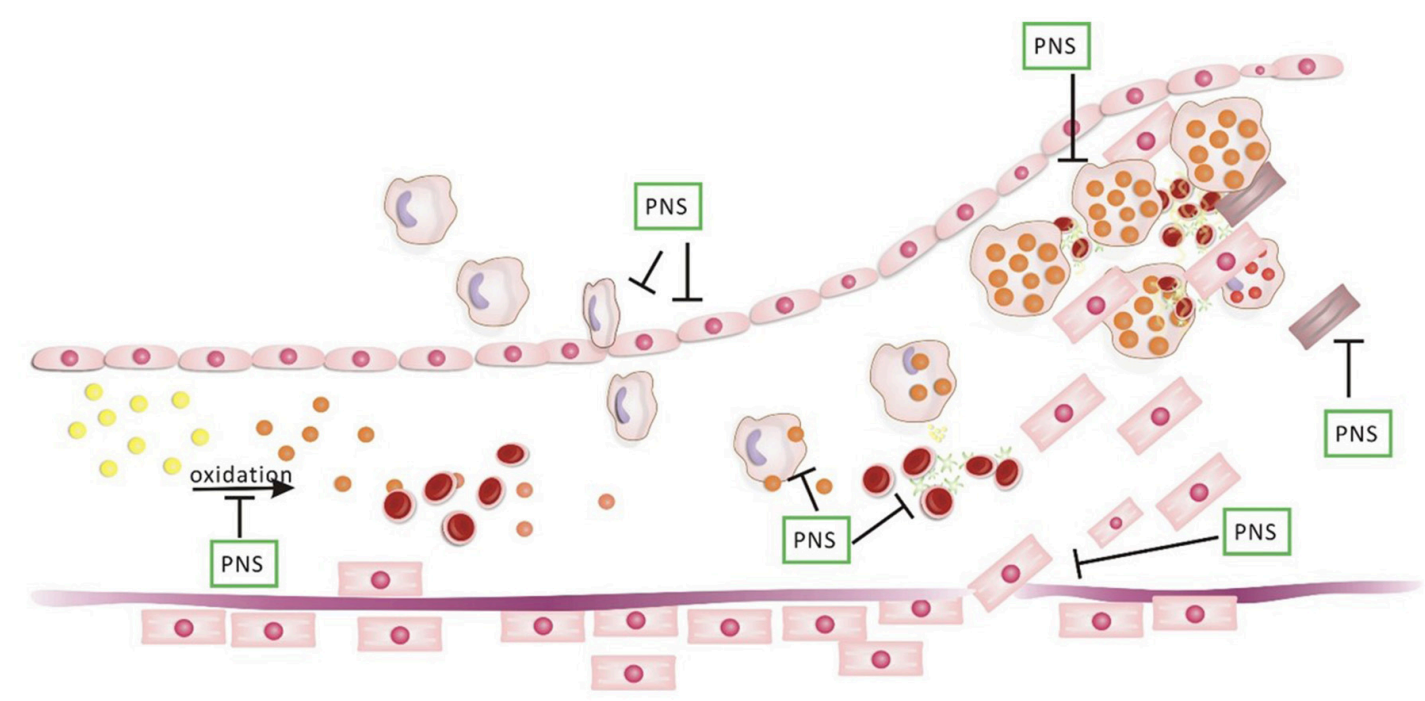

FIGURE 6 | PNS on the evolution of atherosclerotic plaque. In the evolution of atherosclerosis plaque, PNS has effects on the oxidation of LDL, the accumulation of lipoprotein, chemoattractant cytokines related to macrophages, modified lipoprotein particles, platelet aggregation, the migration of smooth muscle cells (SMCs), apoptosis of SMCs and the development of foam cells.

different mechanisms with nitroglycerin. PNS was recommended when patients can't tolerate the side effects of nitroglycerin such as headache, dizziness, tachycardia. Patients with nitrate resistance were also recommended to administrate PNS.

Inflammation runs through the initiation, formation and onset of CAD. The widespread presence of inflammation generates the major feature of vulnerable plaques (Crea and Liuzzo, 2013). PNS exerts anti-inflammation by several signaling pathways. PNS can regulate pro-inflammatory factors by inhibiting ROS, TNA alpha, NF- $\mathrm{B}$, and promoting SOD. Interestingly, PNS has dual functions on COX-2 at different periods. An individual PNS, NR1 can suppress inflammatory cytokines production by activating PPAR gamma and by suppressing ERK and $\mathrm{PKB}$, inhibiting TNF-alpha in vitro and in vivo. Inflammation plays an important role in the whole process of CAD, PNS with less side effects could be administered for a long time. Meanwhile, long-term application of PNS could reduce the end point of CAD.

Different with western medicines, TCM acts on several targets to play a variety of roles on the mechanisms of the disease. PNS has effects simultaneously on anti-inflammation, the regulation of lipid metabolism and the coagulation system, anti-apoptosis, pro-angiogenesis, anti-atherosclerosis, and antimyocardial ischaemia. In the entire pathological process of $\mathrm{CAD}$, at different pathological stages, PNS can effectively reduce the occurrence and the development of CAD. In several RCTs, PNS effectively reduce the end-point of CAD, greatly regulate the lipids, improve performance on the ECG and reduce the frequency and the duration of angina attacks. Thus, PNS is a potential agent against CAD.

Recently, the medical community has gradually assigned importance to the primary prevention of CAD with PNS due to their unique advantages. In primary prevention, PNS can regulate lipid metabolism and hypertension (Pan et al., 2012) and inhibit platelet aggregation. Aspirin has side effects such as gastrointestinal reactions and statins can cause liver injury. Especially in preventive treatment, long-term administration will increase the rate of side effects. And PNS appear to be safer. PNS have multiple targets, a wide range of therapeutic effects and high safety. Therefore, we believe that it has great potential in the treatment of CAD.

\section{Limitations and Perspectives}

Currently, increasing research is focusing on individual PNS. However, these studies are still rare compared to those on total PNS. Individual PNS could have contradictory functions, especially on platelets. Four main ginsenosides (Rg1, Re, NR1, and Rg2) that exist in PNS also showed anti-platelet and anticoagulation activity. Both Rg1 and Rg2 could significantly extend blood clotting time. However, notoginsenoside Ft1 was procoagulant and induced dose-dependent platelet aggregation. Therefore, PNS could be further separated in order to thoroughly investigate the function of $P$. notoginseng. In addition, the mechanism of PNS in CAD is complicated, so the work of individual PNS multi-target networks will further raise the potential of $P$. notoginseng for the effective treatment of CAD.

In addition, we need to further improve the drug purity and screen concentrations to reveal and enhance the medicinal value of PNS as an individual lipid-lowering drug or an antiplatelet agglutination drug. We have found some research that focuses on comparing PNS and aspirin or PNS and statins. The inhibitory effect of PNS on platelet activation was similar to aspirin, but the inhibitory effect of PNS on platelet adhesion to ECs was superior to aspirin (Wang M. M. et al., 2016). In a RCT OF PNS group, aspirin group and PNS plus aspirin group, the results showed decreased D-dimer, platelet aggregation time, increased international standardization ratio of prothrombin time and prolonged prothrombin time in three groups. Compared with 
PNS, aspirin was more effective than PNS in improving platelet aggregation (Yan et al., 2015). The purity and concentrate increase probably generate stronger effects on CAD.

However, the medical technology and the related animal experiments and RCTs are limited. Moreover, only one 1-year RCT has reported the effect of PNS on the end-point of CAD. However, we hope that some multi-center, large-sample RCTs will provide high-level evidence for the effectiveness of PNS in CAD.

\section{CONCLUSION}

PNS have multiple positive effects in the key processes of CAD, including anti-inflammation, the regulation of lipid metabolism and the coagulation system, anti-apoptosis, pro-angiogenesis, anti-atherosclerosis, and anti-myocardial ischaemia. Long-term use of PNS can effectively reduce the end point of CAD and improve angina pectoris, ECG and lipid metabolism which illustrates that PNS is potential agent on CAD. However, more

\section{REFERENCES}

Aronoff, D. M., Canetti, C., and Peters-Golden, M. (2004). Prostaglandin E2 inhibits alveolar macrophage phagocytosis through an E-prostanoid 2 receptormediated increase in intracellular cyclic AMP. J. Immunol. 173, 559-565. doi: 10.4049/jimmunol.173.1.559

Baigent, C., Keech, A., Kearney, P. M., Blackwell, L., Buck, G., Pollicino, C., et al. (2005). Efficacy and safety of cholesterol-lowering treatment: prospective metaanalysis of data from 90056 participants in 14 randomised trials of statins. Lancet 366, 1267-1278. doi: 10.1016/S0140-6736(05)67394-1

Baker, R. G., Hayden, M. S., and Ghosh, S. (2011). NF-kappaB, inflammation, and metabolic disease. Cell Metab. 13, 11-22. doi: 10.1016/j.cmet.2010.12.008

Barnes, P. M., Bloom, B., and Nahin, R. L. (2008). Complementary and alternative medicine use among adults and children: United States, 2007. Natl. Health Stat. Rep. 1-23.

Blanes, M. G., Oubaha, M., Rautureau, Y., and Gratton, J.-P. (2007). Phosphorylation of tyrosine 801 of vascular endothelial growth factor receptor2 is necessary for Akt-dependent endothelial nitric-oxide synthase activation and nitric oxide release from endothelial cells. J. Biol. Chem. 282, 10660-10669. doi: 10.1074/jbc.M609048200

Capettini, L. S., Cortes, S. F., and Lemos, V. S. (2010). Relative contribution of eNOS and nNOS to endothelium-dependent vasodilation in the mouse aorta. Eur. J. Pharmacol. 643, 260-266. doi: 10.1016/j.ejphar.2010.06.066

Chen, S. W., Li, X. H., Ye, K. H., Jiang, Z. F., and Ren, X. D. (2004). Total saponins of Panax notoginseng protected rabbit iliac artery against balloon endothelial denudation injury. Acta Pharmacol. Sin. 25, 1151-1156.

Chen, S., Liu, J., Liu, X., Fu, Y., Zhang, M., Lin, Q., et al. (2011). Panax notoginseng saponins inhibit ischemia-induced apoptosis by activating PI3K/Akt pathway in cardiomyocytes. J. Ethnopharmacol. 137, 263-270. doi: 10.1016/j.jep.2011.05.011

Chew, D. P., Scott, I. A., Cullen, L., French, J. K., Briffa, T. G., Tideman, P. A., et al. (2016). National Heart Foundation of Australia \& Cardiac Society of Australia and New Zealand: Australian Clinical Guidelines for the Management of Acute Coronary Syndromes 2016. Heart Lung Circ. 25, 895-951. doi: 10.1016/j.hlc.2016.06.789

Chong, A. Y., Caine, G. J., and Lip, G. Y. (2004). Angiopoietin/tie-2 as mediators of angiogenesis: a role in congestive heart failure? Eur. J. Clin. Invest. 34, 9-13. doi: 10.1111/j.1365-2362.2004.01284.x

Christoffolete, M. A., Doleschall, M., Egri, P., Liposits, Z., Zavacki, A. M., Bianco, A. C., et al. (2010). Regulation of thyroid hormone activation via the liver X-receptor/retinoid X-receptor pathway. J. Endocrinol. 205, 179-186. doi: 10.1677/JOE-09-0448 high-level RCTs are expected to provide evidence for the efficacy of PNS in CAD.

\section{AUTHOR CONTRIBUTIONS}

JW and LD designed the work of review; LD, XX, and JH reviewed the literature available on this topic and wrote the paper; XX, YL, and JL contributed in the scientific writing of the manuscript; JW and XX revised the manuscript.

\section{FUNDING}

This work was supported by the National Natural Science Foundation of China (No. 81473561).

\section{ACKNOWLEDGMENTS}

The authors apologize to colleagues whose work was not cited due to space limitations or our oversight.

Crea, F., and Liuzzo, G. (2013). Pathogenesis of acute coronary syndromes. J. Am Coll. Cardiol. 61, 1-11. doi: 10.1016/j.jacc.2012.07.064

Deng, Y., Zhang, T., Teng, F., Li, D., Xu, F., Cho, K., et al. (2015). Ginsenoside Rg1 and Rb1, in combination with salvianolic acid B, play different roles in myocardial infarction in rats. J. Chin. Med. Assoc. 78, 114-120. doi: 10.1016/j.jcma.2014.10.001

Department of Cardiology in Second Affiliated Hospital of Wuhan Medical College (1972). Clinical and Experimental Study on the Treatment of Angina Pectoris by Ginseng. J. Med. Sci. Technol. 20-21.

Dilidar, M. Y. (2009). Resistance and prevention of nitrate drugs. Chin. J. Cardiol. $14,83-85$.

Ding, R.-B., Tian, K., Cao, Y.-W., Bao, J.-L., Wang, M., He, C., et al. (2015). Protective effect of panax notoginseng saponins on acute ethanol-induced liver injury is associated with ameliorating hepatic lipid accumulation and reducing ethanol-mediated oxidative stress. J. Agric. Food Chem. 63, 2413-2422. doi: 10.1021/jf502990n

Dorsam, R. T., and Kunapuli, S. P. (2004). Central role of the P2Y12 receptor in platelet activation. J. Clin. Invest. 113, 340-345. doi: 10.1172/JCI20986

Dou, L., Lu, Y., Shen, T., Huang, X., Man, Y., Wang, S., et al. (2012). Panax notogingseng saponins suppress RAGE/MAPK signaling and NFkappaB activation in apolipoprotein-E-deficient atherosclerosis-prone mice. Cell. Physiol. Biochem. 29, 875-882. doi: 10.1159/000315061

$\mathrm{Du}, \mathrm{Z}$., and Chen, G. (2009). Effects of xuesaitong soft capsule on plasma endothelin and c-reactive protein in patients with Angina Pectoris. Chin. J. Modern Med. 4, 140-141.

Editorial Board of Flora of China, M. (1978). Flora of China. Beijing: Science Publishing House.

Fan, J. S., Liu, D. N., Huang, G., Xu, Z. Z., Jia, Y., Zhang, H. G., et al. (2012). Panax notoginseng saponins attenuate atherosclerosis via reciprocal regulation of lipid metabolism and inflammation by inducing liver $\mathrm{X}$ receptor alpha expression. J. Ethnopharmacol. 142, 732-738. doi: 10.1016/j.jep.2012.05.053

Fan, J.-Z., Wang, Y., Meng, Y., Li, G.-W., Chang, S.-X., Nian, H., et al. (2015). Panax notoginseng saponins mitigate ovariectomy-induced bone loss and inhibit marrow adiposity in rats. Menopause J. North Am. Menopause Soc. 22, 1343-1350. doi: 10.1097/GME.0000000000000471

Feng, S., Feng, S., and Liu, J. (2016). Effects of total saponins of panax notoginseng and atorvastatin on blood lipid, common carotid artery thickness and plaque score in patients with coronary heart disease. Chin. Peoples Med. Educ. 28, 51-53.

Fu, J. H., Li, X. Z., Shang, X. H., and Liu, J. X. (2006). [Protective effects of saponines of stem and leaf of Panax notoginseng on acute myocardial ischemia in anaesthetic dogs]. Zhongguo Zhong Yao Za Zhi 31, 62-65. 
Gao, B., Huang, L., Liu, H., Wu, H., Zhang, E., Yang, L., et al. (2014). Platelet P2Y(1)(2) receptors are involved in the haemostatic effect of notoginsenoside Ft1, a saponin isolated from Panax notoginseng. Br. J. Pharmacol. 171, 214-223. doi: $10.1111 /$ bph. 12435

Gao, L., Zhao, H., Liu, Q., Song, J., Xu, C., Liu, P., et al. (2012). Improvement of hematoma absorption and neurological function in patients with acute intracerebral hemorrhage treated with Xueshuantong. J. Neurol. Sci. 323, 236-240. doi: 10.1016/j.jns.2012.09.028

Gerits, N., Kostenko, S., and Moens, U. (2007). In vivo functions of mitogenactivated protein kinases: conclusions from knock-in and knock-out mice. Trans. Res. 16, 281-314. doi: 10.1007/s11248-006-9052-0

Giacca, M., and Zacchigna, S. (2012). VEGF gene therapy: therapeutic angiogenesis in the clinic and beyond. Gene Ther. 19, 622-629. doi: 10.1038/gt.2012.17

Gong, W., Xiao, Y., Zhang, M., Wang, Y., and Wang, Y. (2013). [Synergistic protective effects of salvianolic acids and Panax notoginseng saponins on cardiomyocytes with hypoxia-reoxygenation injury]. Zhongguo Zhong Yao Za Zhi 38, 1046-1051.

Gu, L., Chen, T., Zhang, Y., and Hou, S. (2015). Research progress on the research of Panax notoginseng polysaccharides [J]. West China J. Pharm. Sci. $30,117-119$.

Guo, J. W., Deng, Z. J., Fu, Y. H., Yang, M., Ren, B., Pan, J. Q., et al. (2009). [Effects of Panax notoginsenoside on TNF-alpha and MMP-2 expressions in rats with post-myocardial infarction ventricular remodeling and the mechanism]. Nan Fang Yi Ke Da Xue Xue Bao. 29, 2048-2050.

Guo, J. W., Li, L. M., Qiu, G. Q., Deng, Z. J., Fu, Y. H., Yang, M., et al. (2010). [Effects of Panax notoginseng saponins on ACE2 and TNF-alpha in rats with post-myocardial infarction-ventricular remodeling]. Zhong Yao Cai. 33, 89-92.

Hall, A. M., Wiczer, B. M., Herrmann, T., Stremmel, W., and Bernlohr, D. A. (2005). Enzymatic properties of purified murine fatty acid transport protein 4 and analysis of acyl-CoA synthetase activities in tissues from FATP4 null mice. J. Biol. Chem. 280, 11948-11954. doi: 10.1074/jbc.M412629200

Han, Y. (2008). Xuesaitong Soft Capsule on the Intervention of Postoperative Intervention in Patients with Coronary Heart Disease. Guangzhou University of Traditional Chinese Medicine

Hansson, G. K. (2005). Mechanisms of disease - Inflammation, atherosclerosis, and coronary artery disease. N. Engl. J. Med. 352, 1685-1695. doi: 10.1056/NEJMra043430

Harris, M. F., Parker, S. M., Litt, J., van Driel, M., Russell, G., Mazza, D., et al. (2015). Implementing guidelines to routinely prevent chronic vascular disease in primary care: the preventive evidence into practice cluster randomised controlled trial. BMJ Open 5:e009397. doi: 10.1136/bmjopen-2015-009397

Hausenloy, D. J., Tsang, A., Mocanu, M. M., and Yellon, D. M. (2005). Ischemic preconditioning protects by activating prosurvival kinases at reperfusion. Am. J. Physiol. 288, H971-H976. doi: 10.1152/ajpheart.00374.2004

Heeren, J., Beisiegel, U., and Grewal, T. (2006). Apolipoprotein E recycling implications for dyslipidemia and atherosclerosis. Arterioscler. Thromb. Vasc. Biol. 26, 442-448. doi: 10.1161/01.ATV.0000201282.64751.47

Helgason, C. M., Bolin, K. M., Hoff, J. A., Winkler, S. R., Mangat, A., Tortorice, K. L., et al. (1994). Development of aspirin resistance in persons with previous ischemic stroke. Stroke 25, 2331-2336. doi: 10.1161/01.STR.25.12.2331

Heslop, C. L., Frohlich, J. J., and Hill, J. S. (2010). Myeloperoxidase and C-reactive protein have combined utility for long-term prediction of cardiovascular mortality after coronary angiography. J. Am. Coll. Cardiol. 55, 1102-1109. doi: 10.1016/j.jacc.2009.11.050

Hirsh, J., Dalen, J. E., Fuster, V., Harker, L. B., Patrono, C., and Roth, G. (1995). Aspirin and other platelet-active drugs. The relationship among dose, effectiveness, and side effects. Chest 108(4 Suppl.), 247S-257S. doi: 10.1378/chest.108.4_Supplement.247S

HKCMMS (2017). Hong Kong Chinese Materia Medica Standards (HKCMMS). Available online at: http://www.cmd.gov.hk/html/b5/hkcmms/cmmlist.html

Hong, S. J., Wan, J. B., Zhang, Y., Hu, G., Lin, H. C., Seto, S. W., et al. (2009). Angiogenic effect of saponin extract from Panax notoginseng on HUVECs in vitro and zebrafish in vivo. Phytother. Res. 23, 677-686. doi: 10.1002/ptr.2705

Hopkins, P. N. (2013). Molecular biology of atherosclerosis. Physiol. Rev. 93, 1317-1542. doi: 10.1152/physrev.00004.2012

Hotamisligil, G. S. (2006). Inflammation and metabolic disorders. Nature 444, 860-867. doi: 10.1038/nature05485

Hou, F. (2016). Clinical observation of 84 Cases of Unstable Angina Pectoris Treated by Xuesaitong Soft Capsule. Chin. Health Nutr. 227.
Huss, J. M., Torra, I. P., Staels, B., Giguère, V., and Kelly, D. P. (2004). Estrogen-related receptor $\alpha$ directs peroxisome proliferatoractivated receptor $\alpha$ signaling in the transcriptional control of energy metabolism in cardiac and skeletal muscle. Mol. Cell Biol. 24, 9079-9091. doi: 10.1128/MCB.24.20.9079-9091.2004

Jellinger, P. S., Handelsman, Y., Rosenblit, P. D., Bloomgarden, Z. T., Fonseca, V. A., Garber, A. J., et al. (2017). AMERICAN ASSOCIATION OF CLINICAL ENDOCRINOLOGISTS AND AMERICAN COLLEGE OF ENDOCRINOLOGY GUIDELINES FOR MANAGEMENT OF DYSLIPIDEMIA AND PREVENTION OF CARDIOVASCULAR DISEASE - EXECUTIVE SUMMARYComplete Appendix to Guidelines available at http://journals.aace.com. Endocrine Pract. 23, 479-497. doi: 10.4158/EP171764.GL

Jia, Y., Li, Z. Y., Zhang, H. G., Li, H. B., Liu, Y., and Li, X. H. (2010). Panax notoginseng saponins decrease cholesterol ester via up-regulating ATPbinding cassette transporter A1 in foam cells. J. Ethnopharmacol. 132, 297-302. doi: $10.1016 /$ j.jep.2010.08.033

Kim, D. H. (2012). Chemical Diversity of Panax ginseng, Panax quinquifolium, and Panax notoginseng. J. Ginseng. Res. 36, 1-15. doi: 10.5142/jgr.2012.36.1.1

Kong, Y., and Zhang, H. (2006). Clinical Observation of 52 Cases of Coronary Heart Disease with Unstable Angina Pectoris Treated by Xuesaitong Soft Capsule. Yunnan Trad. Chin.Med.J. 24.

Kuang, G., Huang, Z., Bai, C., Hu, Y. Q., and Dai, T. (2011). Effect of xuesaitong soft capsule on efficacy of unstable angina pectoris and ECG QT dispersion. Lingnan J. Emerg. Med. 16, 455-456.

Labreuche, J., Deplanque, D., Touboul, P.-J., Bruckert, E., and Amarenco, P. (2010). Association between change in plasma triglyceride levels and risk of stroke and carotid atherosclerosis: systematic review and meta-regression analysis. Atherosclerosis 212, 9-15 doi: 10.1016/j.atherosclerosis.2010. 02.011

Levine, G. N., Bates, E. R., Bittl, J. A., Brindis, R. G., Fihn, S. D., Fleisher, L. A., et al. (2016). ACC/AHA Guideline Focused Update on Duration of Dual Antiplatelet Therapy in Patients with Coronary Artery Disease. J. Am. Coll. Cardiol. 68, 1082-1115. doi: 10.1016/j.jacc.2016.03.513

Li, C. T., Wang, H. B., and Xu, B. J. (2013). A comparative study on anticoagulant activities of three Chinese herbal medicines from the genus Panax and anticoagulant activities of ginsenosides Rg1 and Rg2. Pharm. Biol. 51, 1077-1080. doi: 10.3109/13880209.2013.775164

Li, J., Xie, Z. Z., Tang, Y. B., Zhou, J. G., and Guan, Y. Y. (2011). Ginsenoside$\mathrm{Rd}$, a purified component from panax notoginseng saponins, prevents atherosclerosis in apoE knockout mice. Eur. J. Pharmacol. 652, 104-110. doi: 10.1016/j.ejphar.2010.11.017

Li, J., Yang, L., Wang, J. Z., Zhang, C. C., Wang, T., Liu, Z. Q., et al. (2014). [Study on protective effect of Panax notoginseng total saponins on H9c2 cells senescence against D-galactose]. Zhong Yao Cai 37, 1421-1424.

Li, S. Y., Wang, X. G., Ma, M. M., Liu, Y., Du, Y. H., Lv, X. F., et al. (2012). Ginsenoside-Rd potentiates apoptosis induced by hydrogen peroxide in basilar artery smooth muscle cells through the mitochondrial pathway. Apoptosis 17, 113-120. doi: 10.1007/s10495-011-0671-4

Liu, G., Wang, B., Zhang, J., Jiang, H., and Liu, F. (2009). Total panax notoginsenosides prevent atherosclerosis in apolipoprotein E-knockout mice: Role of downregulation of CD40 and MMP-9 expression. J. Ethnopharmacol. 126, 350-354. doi: 10.1016/j.jep.2009.08.014

Liu, H. Z., Liu, Z. L., Zhao, S. P., Sun, C. Z., and Yang, M. S. (2014). Protective mechanism of Panax notoginseng saponins on rat hemorrhagic shock model in recovery stage. Cell Biochem. Biophys. 70, 1719-1724. doi: 10.1007/s12013-014-0119-x

Liu, J., Wang, Y., Qiu, L., Yu, Y., and Wang, C. (2014). Saponins of Panax notoginseng: chemistry, cellular targets and therapeutic opportunities in cardiovascular diseases. Expert Opin. Invest. Drugs 23, 523-539. doi: $10.1517 / 13543784.2014 .892582$

Liu, L., Zhu, L., Zou, Y., Liu, W., Zhang, X., Wei, X., et al. (2014). Panax notoginseng saponins promotes stroke recovery by influencing expression of Nogo-A, NgR and p75NGF, in vitro and in vivo. Biol. Pharm. Bull. 37, 560-568. doi: 10.1248/bpb.b13-00770

Liu, X., Li, J., Yang, G., and Wang, J. (2008). Effect of active components of activating blood circulation on inflammatory level of patients with unstable angina pectoris and blood stasis syndrome. Chin. J. Trad. Chin. Med. 33, 2950-2953. 
Liu, Y., Hao, F., Zhang, H., Cao, D., Lu, X., and Li, X. (2013). Panax notoginseng saponins promote endothelial progenitor cell mobilization and attenuate atherosclerotic lesions in apolipoprotein E knockout mice. Cell Physiol. Biochem. 32, 814-826. doi: 10.1159/000354484

Liu, Y., Zhang, H. G., Jia, Y., and Li, X. H. (2010). Panax notoginseng saponins attenuate atherogenesis accelerated by zymosan in rabbits. Biol. Pharm. Bull. 33, 1324-1330. doi: 10.1248/bpb.33.1324

Madrazo, J. A., and Kelly, D. P. (2008). The PPAR trio: regulators of myocardial energy metabolism in health and disease. J. Mol. Cell Cardiol. 44, 968-975. doi: 10.1016/j.yjmcc.2008.03.021

Mann, D. L., Zipes, D., Libby, P., and Bonow, R. O. (2014). Braunwald's Heart Disease. Boston, MA: Elsevier.

Marks, F., Klingmuller, U., and Muller-Decker, K. (2009). Cellular Signal Processing. An Introduction to the Molecular Mechanisms of Signal Transduction. NewYork, NY: Garland Science, Taylor and Francis Group.

McBane, R. D. I. I., Hardison, R. M., and Sobel, B. E. (2010). BARI 2D Study Group. Comparison of plasminogen activator inhibitor-1, tissue type plasminogen activator antigen, fibrinogen, and D-dimer levels in various age decades in patients with type 2 diabetes mellitus and stable coronary artery disease (from the BARI 2D trial). Am. J. Cardiol. 105, 17-24. doi: 10.1016/j.amjcard.2009.08.643

Meir, K. S., and Leitersdorf, E. (2004). Atherosclerosis in the apolipoprotein Edeficient mouse - A decade of progress. Arterioscler. Thromb. Vasc. Biol. 24, 1006-1014. doi: 10.1161/01.ATV.0000128849.12617.f4

Meng, K., Zhu, H., Song, X., Ge, C., Zhou Y., Dai J., et al. (2013). Effects of Panax Notoginseng saponins combined with double antiplatelet therapy on cardiovascular events in patients with coronary artery stent. Chin. Med. 8, $140-141$

Mitsos, S., Katsanos, K., Koletsis, E., Kagadis, G. C., Anastasiou, N., Diamantopoulos, A., et al. (2012). Therapeutic angiogenesis for myocardial ischemia revisited: basic biological concepts and focus on latest clinical trials. Angiogenesis 15, 1-22. doi: 10.1007/s10456-011-9240-2

Mozaffarian, D., Benjamin, E. J., Go, A. S., Arnett, D. K., Blaha, M. J., Cushman, M., et al. (2016). Executive Summary: Heart Disease and Stroke Statistics2016 Update A Report From the American Heart Association. Circulation 133, 447-454. doi: 10.1161/CIR.0000000000000366

Münzel, T., Daiber, A., and Gori, T. (2011). Nitrate therapy. Circulation 123:2132. doi: 10.1161/CIRCULATIONAHA.110.981407

Nording, H. M., Seizer, P., and Langer, H. F. (2015). Platelets in inflammation and atherogenesis. Front. Immunol. 6:98. doi: 10.3389/fimmu.2015.00098

Nylander, S., and Schulz, R. (2016). Effects of P2Y12 receptor antagonists beyond platelet inhibition-comparison of ticagrelor with thienopyridines. $\mathrm{Br}$. J. Pharmacol. 173, 1163-1178. doi: 10.1111/bph.13429

Nyyssonen, K., Kurl, S., Karppi, J., Nurmi, T., Baldassarre, D., Veglia, F., et al. (2012). LDL oxidative modification and carotid atherosclerosis: results of a multicenter study. Atherosclerosis 225, 231-236. doi: 10.1016/j.atherosclerosis.2012.08.030

O'Gara, P. T., Kushner, F. G., Ascheim, D. D., Casey, D. E., Chung, M. K., de Lemos, J. A., et al. (2013). ACCF/AHA Guideline for the Management of ST-Elevation Myocardial Infarction. Circulation 127:e362-e425. doi: 10.1161/CIR.0b013e3182742cf6

Ohno, M., Takemura, G., Ohno, A., Misao, J., Hayakawa, Y., Minatoguchi, S., et al. (1998). "Apoptotic" myocytes in infarct area in rabbit hearts may be oncotic myocytes with DNA fragmentation: analysis by immunogold electron microscopy combined with in situ nick end-labeling. Circulation 98, 1422-1430. doi: 10.1161/01.CIR.98.14.1422

Oliveira-Marques, V., Marinho, H. S., Cyrne, L., and Antunes, F. (2009). Role of hydrogen peroxide in NF-kappaB activation: from inducer to modulator. Antioxid. Redox Signal. 11, 2223-2243. doi: 10.1089/ars.2009.2601

Pan, C., Huo, Y., An, X., Singh, G., Chen, M., Yang, Z., et al. (2012). Panax notoginseng and its components decreased hypertension via stimulation of endothelial-dependent vessel dilatation. Vasc. Pharmacol. 56, 150-158. doi: 10.1016/j.vph.2011.12.006

Patel, J. V., Lim, H. S., Nadar, S., Tayebjee, M., Hughes, E. A., and Lip, G. Y. (2006). Abnormal soluble CD40 ligand and C-reactive protein concentrations in hypertension: relationship to indices of angiogenesis. J. Hypertens. 24, 117-121. doi: 10.1097/01.hih.0000198034.09010.bc

Qi, H., Huang, Y., Yang, Y., Dou, G., Wan, F., Zhang, W., et al. (2016). Anti-platelet activity of panaxatriol saponins is mediated by suppression of intracellular calcium mobilization and ERK2/p38 activation. BMC Complement. Altern. Med. 16:174. doi: 10.1186/s12906-016-1160-7

Qi, S., Xin, Y., Guo, Y., Diao, Y., Kou, X., Luo, L., et al. (2012). Ampelopsin reduces endotoxic inflammation via repressing ROS-mediated activation of PI3K/Akt/NF-kappa B signaling pathways. Int. Immunopharmacol. 12, 278-287. doi: 10.1016/j.intimp.2011.12.001

Santos-Gallego, C. G., Picatoste, B., and Badimon, J. J. (2014). Pathophysiology of acute coronary syndrome. Curr. Atheroscler. Rep. 16:401. doi: 10.1007/s11883-014-0401-9

Serebruany, V. L., Steinhubl, S. R., Berger, P. B., Malinin, A. I., Bhatt, D. L., and Topol, E. J. (2005). Variability in platelet responsiveness to clopidogrel among 544 individuals. J. Am. Coll. Cardiol. 45, 246-251 doi: 10.1016/j.jacc.2004.09.067

Shang, Q., Xu, H., Liu, Z., Chen, K., and Liu, J. (2013). Oral Panax notoginseng preparation for coronary heart disease: a systematic review of randomized controlled trials. Evid. Based Complement. Altern. Med. 2013:940125. doi: 10.1155/2013/940125

Shen, K., Leung, S. W., Ji, L., Huang, Y., Hou, M., Xu, A., et al. (2014). Notoginsenoside Ft1 activates both glucocorticoid and estrogen receptors to induce endothelium-dependent, nitric oxide-mediated relaxations in rat mesenteric arteries. Biochem. Pharmacol. 88, 66-74. doi: 10.1016/j.bcp.2014.01.007

Smith, S. C. Jr., Benjamin, E. J., Bonow, R. O., Braun, L. T., Creager, M. A., Franklin, B. A., et al. (2011). AHA/ACCF secondary prevention and risk reduction therapy for patients with coronary and other atherosclerotic vascular disease: 2011 update: a guideline from the American Heart Association and American College of Cardiology Foundation. Circulation 124, 2458-2473. doi: 10.1161/CIR.0b013e318235eb4d

Song, W., Wang, Z., and Zengxianyi. (2005). Clinical observation of coronary heart disease and angina pectoris treated by xuesaitong soft capsule. Chin. J. Trad. Chin. Med. 14, 707-708.

Su, P., Du, S., Li, H., Li, Z., Xin, W., and Zhang, W. (2016). Notoginsenoside R1 inhibits oxidized low-density lipoprotein induced inflammatory cytokines production in human endothelial EA.hy926 cells. Eur. J. Pharmacol. 770, 9-15. doi: 10.1016/j.ejphar.2015.11.040

Sun, B., Xiao, J., Sun, X. B., and Wu, Y. (2013). Notoginsenoside R1 attenuates cardiac dysfunction in endotoxemic mice: an insight into oestrogen receptor activation and PI3K/Akt signalling. Br. J. Pharmacol. 168, 1758-1770. doi: 10.1111/bph.12063

Sun, X., Gao, R. L., Lin, X. J., Xu, W. H., and Chen, X. H. (2013). Panax notoginseng saponins induced up-regulation, phosphorylation and binding activity of MEK, ERK, AKT, PI-3K protein kinases and GATA transcription factors in hematopoietic cells. Chin. J. Integr. Med. 19, 112-118. doi: 10.1007/s11655-012-1306-4

Tang, Z. S., Guo, L. W., Wang, B., and Song, Y. P. (2010). [Optimization of PNS/TGCO components effect on experimental Qi stagnation and blood stasis type of coronary heart disease with increase-decrease baseline geometric proportion design method]. Zhong Yao Cai. 33, 1439-1442.

Tello-Montoliu, A., Patel, J. V., and Lip, G. Y. (2006). Angiogenin: a review of the pathophysiology and potential clinical applications. J. Thromb. Haemost. 4, 1864-1874. doi: 10.1111/j.1538-7836.2006.01995.x

Teng, F. (2014). PNS on the Pathogenesis of Coronary Heart Disease and Blood Stasis Associated with miRNA and Target Genes. Chinese Academy of Traditional Chinese Medicine.

Thompson, P. D., Panza, G., Zaleski, A., and Taylor, B. (2016). Statin-associated side effects. J. Am. Coll. Cardiol. 67, 2395-2410. doi: 10.1016/j.jacc.2016.02.071

Van Raamsdonk, J. M., and Hekimi, S. (2012). Superoxide dismutase is dispensable for normal animal lifespan. Proc. Natl. Acad. Sci. U.S.A. 109, 5785-5790. doi: 10.1073/pnas.1116158109

Venuraju, S. M., Yerramasu, A., Corder, R., and Lahiri, A. (2010). Osteoprotegerin as a predictor of coronary artery disease and cardiovascular mortality and morbidity. J. Am. Coll. Cardiol. 55, 2049-2061. doi: 10.1016/j.jacc.2010. 03.013

Wan, X. F. (2011). Effect of Xuesaitong Soft Capsule on Unstable Angina Pectoris. Pract. J. Cardio Pulmon. Hypertens. 1768.

Wang, J., Wu, L., Zhang, W., and Deng, C. (2009). [Effect of Panax notoginseng saponins on vascular intima hyperplasia and PCNA expression in rat aorta after balloon angioplasty]. Zhongguo Zhong Yao Za Zhi 34, 735-739. 
Wang, J., Xu, J., and Zhong, J. B. (2004). [Effect of Radix notoginseng saponins on platelet activating molecule expression and aggregation in patients with blood hyperviscosity syndrome]. Zhongguo Zhong Xi Yi Jie He Za Zhi 24, 312-316.

Wang, L.-C., Zhang, W.-S., Liu, Q., Li, J., Alolga, R. N., Liu, K., et al. (2015). A standardized notoginseng extract exerts cardioprotection by attenuating apoptosis under endoplasmic reticulum stress conditions. J. Funct. Foods 16, 20-27. doi: 10.1016/j.jff.2015.04.018

Wang, M. M., Xue, M., Xu, Y. G., Miao, Y., Kou, N., Yang, L., et al. (2016). Panax notoginseng saponin is superior to aspirin in inhibiting platelet adhesion to injured endothelial cells through COX pathway in vitro. Thromb Res. 141, 146-152. doi: 10.1016/j.thromres.2016.03.022

Wang, Q., Li, C., Zhang, Q., Wang, Y., Shi, T., Lu, L., et al. (2016). The effect of Chinese herbs and its effective components on coronary heart disease through PPARs-PGClalpha pathway. BMC Complement. Altern. Med. 16:514. doi: 10.1186/s12906-016-1496-Z

Wang, T., Guo, R., Zhou, G., Zhou, X., Kou, Z., Sui, F., et al. (2016). Traditional uses, botany, phytochemistry, pharmacology and toxicology of Panax notoginseng (Burk.) F.H. Chen: A review. J. Ethnopharmacol. 188, 234-258. doi: 10.1016/j.jep.2016.05.005

Wei, J., and Wang, J. (1987). Research on Flavonoids in Panax notoginseng leaves. Chin. J. Trad. Chin. Med. 11, 33-35.

Wei, Y. (2010). Xuesaitong soft capsule in treating 90 cases of coronary heart disease with unstable angina pectoris. Chin. Modern Drug Appl. 4, 20-21.

WHO (2014). World Health Organization. Global Health Estimates: Deaths by Cause, Age, Sex and Country, 2000-2012. Geneva: WHO.

Wong, A. S. T., Che, C.-M., and Leung, K.-W. (2015). Recent advances in ginseng as cancer therapeutics: a functional and mechanistic overview. Nat. Prod. Rep. 32, 256-272. doi: 10.1039/C4NP00080C

Wu, M.-Z. (1979). Study on saponin composition of Yunnan-producing plants [J]. Acta Metall. Sin. 1, 119-123.

Xiong, X.-J., Wang, Z., and Wang, J. (2015). Innovative strategy in treating angina pectoris with chinese patent medicines by promoting blood circulation and removing blood stasis: experience from combination therapy in chinese medicine. Curr. Vasc. Pharmacol. 13, 540-553. doi: 10.2174/1570161112666141014153735

Xu, L., Liu, J. T., Liu, N., Lu, P. P., and Pang, X. M. (2011). Effects of Panax notoginseng saponins on proliferation and apoptosis of vascular smooth muscle cells. J. Ethnopharmacol. 137, 226-230. doi: 10.1016/j.jep.2011.05.020

Yan, X., Cui, D., and Yang, Q. (2015). Effects of Panax Notoginseng saponins combined with aspirin on platelet aggregation in elderly patients with coronary heart disease. Shandong Med. J. 55, 47-48.

Yang, B. R., Hong, S. J., Lee, S. M., Cong, W. H., Wan, J. B., Zhang, Z. R., et al. (2016). Pro-angiogenic activity of notoginsenoside R1 in human umbilical vein endothelial cells in vitro and in a chemical-induced blood vessel loss model of zebrafish in vivo. Chin. J. Integr. Med. 22, 420-429. doi: $10.1007 / \mathrm{s} 11655-014-1954-8$

Yang, X., Xiong, X., Wang, H., and Wang, J. (2014). Protective effects of panax notoginseng saponins on cardiovascular diseases: a comprehensive overview of experimental studies. Evid. Based Complement. Alternat. Med. 2014:204840. doi: $10.1155 / 2014 / 204840$

Yang, X., Xiong, X., Wang, H., Yang, G., and Wang, J. (2013). Xuesaitong soft capsule (chinese patent medicine) for the treatment of unstable angina pectoris: a meta-analysis and systematic review. Evid. Based Complement. Altern. Med. 2013:948319. doi: 10.1155/2013/948319

Yao, Y., Wu, W. Y., Guan, S. H., Jiang, B. H., Yang, M., Chen, X. H., et al. (2008a). Proteomic analysis of differential protein expression in rat platelets treated with notoginsengnosides. Phytomedicine 15, 800-807. doi: 10.1016/j.phymed.2008.06.013

Yao, Y., Wu, W. Y., Liu, A. H., Deng, S. S., Bi, K. S., Liu, X., et al. (2008b). Interaction of salvianolic acids and notoginsengnosides in inhibition of ADP-induced platelet aggregation. Am. J. Chin. Med. 36, 313-328. doi: $10.1142 / \mathrm{S} 0192415 \mathrm{X} 08005795$

Yu, D. (2010). Clinical effect of Xuesaitong soft capsule in the treatment of unstable angina pectoris. Clin. Med. Pract. 19, 38-39.

Yuan, Z. B., Zhang, H. G., Jia, Y., Cheng, Y. Q., and Li, X. H. (2009). Temporal expression of cyclooxygenase-2 in peritoneal macrophages of rats and effects of panax notoginseng saponins. Inflamm. Res. 58, 74-80. doi: 10.1007/s00011-009-8044-y

Yuan, Z., Liao, Y., Tian, G., Li, H., Jia, Y., Zhang, H., et al. (2011). Panax notoginseng saponins inhibit Zymosan A induced atherosclerosis by suppressing integrin expression, FAK activation and NF-kappaB translocation. J. Ethnopharmacol. 138, 150-155. doi: 10.1016/j.jep.2011. 08.066

Yue, Q. X., Xie, F. B., Song, X. Y., Wu, W. Y., Jiang, B. H., Guan, S. H., et al. (2012). Proteomic studies on protective effects of salvianolic acids, notoginsengnosides and combination of salvianolic acids and notoginsengnosides against cardiac ischemic-reperfusion injury. J Ethnopharmacol. 141, 659-667. doi: 10.1016/j.jep.2011.08.044

Zakar, H. M., and Ken, F. (2003). Profound negative regulatory effects by resveratrol on vascular smooth muscle cells: a role of p53p21WAF1/CIP1 pathway. Biochem. Biophys. Res. Commun. 311, 546-555. doi: $10.1016 / j . b b r c .2003 .10 .023$

Zhang, F., Kent, K. C., Yamanouchi, D., Zhang, Y., Kato, K., Tsai, S., et al. (2009). Anti-receptor for advanced glycation end products therapies as novel treatment for abdominal aortic aneurysm. Ann. Surg. 250, 416-423. doi: 10.1097/SLA.0b013e3181b41a18

Zhang, H. S., and Wang, S. Q. (2006). Notoginsenoside R1 from Panax notoginseng inhibits TNF-alpha-induced PAI-1 production in human aortic smooth muscle cells. Vasc. Pharmacol. 44, 224-230. doi: 10.1016/j.vph.2005.12.002

Zhang, J.-S., Zhang, B.-X., Du, M.-M., Wang, X.-Y., and Li, W. (2016). Chinese preparation Xuesaitong promotes the mobilization of bone marrow mesenchymal stem cells in rats with cerebral infarction. Neural Regen. Res. 11, 292-297. doi: 10.4103/1673-5374.177738

Zhang, T. (2014). Clinical observation of Xuesaitong Capsule combined with trimetazidine in the treatment of angina pectoris of coronary heart disease. Med. Information 27, 207-208.

Zhang, W., Chen, G., and Deng, C. Q. (2012). Effects and mechanisms of total Panax notoginseng saponins on proliferation of vascular smooth muscle cells with plasma pharmacology method. J. Pharm. Pharmacol. 64, 139-145. doi: $10.1111 / j .2042-7158.2011 .01379 . x$

Zhang, W., Wojta, J., and Binder, B. R. (1994). Effect of notoginsenoside R1 on the synthesis of tissue-type plasminogen activator and plasminogen activator inhibitor-1 in cultured human umbilical vein endothelial cells. Arterioscler. Thromb. 14, 1040-1046. doi: 10.1161/01.ATV.14.7.1040

Zhang, Y. G., Zhang, H. G., Zhang, G. Y., Fan, J. S., Li, X. H., Liu, Y. H., et al. (2008). Panax notoginseng saponins attenuate atherosclerosis in rats by regulating the blood lipid profile and an anti-inflammatory action. Clin. Exp. Pharmacol. Physiol. 35, 1238-1244. doi: 10.1111/j.1440-1681.2008.04997.x

Zheng, L. (2014). Clinical observation of 56 cases of stable angina pectoris treated by xuesaitong. Chin. Foreign Health Digest. 62.

Zhong, L., Zhou, X. L., Liu, Y. S., Wang, Y. M., Ma, F., Guo, B. L., et al. (2015). Estrogen receptor alpha mediates the effects of notoginsenoside $\mathrm{R} 1$ on endotoxin-induced inflammatory and apoptotic responses in H9c2 cardiomyocytes. Mol. Med. Rep. 12, 119-126. doi: 10.3892/mmr.2015.3394

Zhou, H., and Bai, J. (2009). Effect of xuesaitong soft capsule on coronary heart disease and angina pectoris and its effect on plasma endothelin and nitric oxide levels. Chin. J. Pract. Med. 36:82.

Zhu, D., Wu, L., Li, C. R., Wang, X. W., Ma, Y. J., Zhong, Z. Y., et al. (2009). Ginsenoside Rg1 protects rat cardiomyocyte from hypoxia/reoxygenation oxidative injury via antioxidant and intracellular calcium homeostasis. J. Cell Biochem. 108, 117-124. doi: 10.1002/jcb.22233

Conflict of Interest Statement: The authors declare that the research was conducted in the absence of any commercial or financial relationships that could be construed as a potential conflict of interest.

Copyright $\odot 2017$ Duan, Xiong, Hu, Liu, Li and Wang. This is an open-access article distributed under the terms of the Creative Commons Attribution License (CC BY). The use, distribution or reproduction in other forums is permitted, provided the original author(s) or licensor are credited and that the original publication in this journal is cited, in accordance with accepted academic practice. No use, distribution or reproduction is permitted which does not comply with these terms. 